\title{
Active gel segment behaving as an active particle
}

\author{
P. Recho, ${ }^{1, *}$ T. Putelat,,${ }^{2,3, \dagger}$ and L. Truskinovsky ${ }^{4, \sharp}$ \\ ${ }^{1}$ LIPhy, CNRS-UMR 5588, Université Grenoble Alpes, F-38000 Grenoble, France \\ ${ }^{2}$ SAS, Rothamsted Research, Harpenden, AL5 2JQ, United Kingdom \\ ${ }^{3}$ DEM, Queen's School of Engineering, University of Bristol, Bristol, BS8 1TR, United Kingdom \\ ${ }^{4}$ PMMH, CNRS-UMR 7636, ESPCI PSL, F-75005 Paris, France
}

(Received 11 July 2019; revised manuscript received 8 October 2019; published 4 December 2019)

\begin{abstract}
We reduce a one-dimensional model of an active segment (AS), which is used, for instance, in the description of contraction-driven cell motility, to a zero-dimensional model of an active particle (AP) characterized by two internal degrees of freedom: position and polarity. Both models give rise to hysteretic force-velocity relations showing that an active agent can support two opposite polarities under the same external force and that it can maintain the same polarity while being dragged by external forces with opposite orientations. This double bistability results in a rich dynamic repertoire which we illustrate by studying static, stalled, motile, and periodically repolarizing regimes displayed by an active agent confined in a viscoelastic environment. We show that the AS and AP models can be calibrated to generate quantitatively similar dynamic responses.
\end{abstract}

DOI: 10.1103/PhysRevE.100.062403

\section{INTRODUCTION}

Most mammalian cells have a remarkable ability to selfpropel even if confronted by an opposing mechanical force [1]. The implied macroscopic motion is generated microscopically inside the cellular cytoskeleton, an actively cross linked biopolymer meshwork that can, for instance, spontaneously and inhomogeneously contract in response to various external and internal stimuli [2]. In particular, cells are known to adjust their mode of self-propulsion by sensing the gradients of chemokines, detecting the density of ligands and probing the stiffness of the environment [3,4]. The integration of all these cues [5] allows cells to continuously reorganize their cytoskeleton and in this way to actively control their motility mechanism [6,7].

The effect of mechanical stimuli on the dynamics of the cytoskeleton is raising increasing attention [8]. Some cells, like immune or cancer cells, are typically exposed to spatially inhomogeneous rheological environments which may generate time-dependent elastic and viscous resistance when they migrate in an organism. Cellular responses can also be directly linked to the action of external forces as in the case of the various outcomes of cell collision tests $[9,10]$, which has been recently explained mechanically [11] without involving biochemical pathways $[12,13]$. Understanding the response of the cytoskeletal reorganization to mechanical loading $[14,15]$ may also guide the design of microscale bioinspired robots which would then perform various healing functions.

Many important advances have been made in the modeling of the migration of individual cells which involves not only cytoskeletal contraction but also other complex phenomena,

\footnotetext{
*pierre.recho@univ-grenoble-alpes.fr

†t.putelat@bristol.ac.uk

†lev.truskinovsky@espci.fr
}

in particular, active polymerization and active adhesion [16]. All these mechanisms have been successfully captured by the continuum liquid crystal theory with incorporated tensorial chemomechanical coupling [17-22]. However, while being comprehensive, the resulting models mostly rely on largescale numerical simulations. This makes their integration as building blocks of a kinetic theory of tissues $[23,24]$ computationally costly, and simpler models are needed to study the collective behavior of cells [25]. Capturing the mechanical interaction of a cell with its environment at such a reduced level is crucial for the adequate reproduction of the emerging active phases [26,27].

To this end, we condense in this paper a one-dimensional model of an active segment (AS) [28], which is used, for instance, in the description of contraction-driven cell motility on tracks, to a zero-dimensional model of an active particle (AP) characterized by two internal degrees of freedom: position and polarity. By focusing on contraction, we are motivated by the experimental observations that a crucial building block of cell repolarization, which plays an important role in both cell collisions and cell oscillations, is myosin contractility [28,29]. In this exploratory study we limit our consideration to one dimension having in mind that such a setting is close to the classical well-calibrated experimental assays [30] while also carrying some physiological significance: a typical situation of three-dimensional in vivo motility is when cells travel along the fibers of the extracellular matrix.

In contrast to some well-known representations of sizeless active agents [31], the obtained AP model accounts for the temporal dynamics of the degree of cell polarization in the presence of external forces. In particular, we show that both AS and AP models support two coexisting dynamic regimes: frictional, when the active object is dragged by the force, and antifrictional, when it is dragging the cargo. The fact that the system is able to switch from one of these nonequilibrium 
steady states to the other through a hysteresis loop is a clear manifestation of the fact that repolarization can emerge as a result of the direct self-organization of the cytoskeleton in response to a mechanical action without additional biochemical regulation.

In the case of self-propulsion in a viscous environment we find a continuous transition between the static (no polarity) and the motile (two symmetry-related polarities) regimes at a critical activity threshold which becomes viscosity independent at sufficiently large viscosities. In the case of elastic confinement, we identify three dynamic regimes: static (no polarity), stalled (two symmetry-related polarities), and oscillatory (periodically varying polarity). In a certain range of parameters, our theory predicts a metastable coexistence between the stalled and the oscillatory regimes, which opens the possibility of complex stop-and-go dynamics in the presence of noise.

We show that the AS and AP models can be calibrated to generate not only qualitatively but also quantitatively similar dynamic responses. This is rather remarkable in view of the fact that the AS model is described mathematically by a free boundary problem formulated for nonlinear partial differential equations of Keller-Segel type while the AP model ultimately reduces to a single ordinary differential equation.

The paper is organized as follows. In Sec. II we present the AS model accounting for the presence of a general external force field. In Sec. III we formally reduce this model to a set of two ordinary differential equations describing a sizeless active particle and specify the calibration procedure for the reduced model. In Sec. IV we compare the velocity-force relation obtained in AS and AP models and show that they can be made quantitatively similar. Then in Sec. V we study the dynamics of an AP subjected to a viscous force. The case of an AP attached to fixed wall through a linearly elastic spring is studied in Sec. VI. Section VII summarizes our results.

\section{THE ACTIVE SEGMENT (AS)}

In this section we review the model of an active gel segment performing a contraction-driven crawling on a rigid surface $[1,28,32]$. Our focus is on the unexplored role of the distributed external forces in this context.

\section{A. The active gel model}

A cell crawling on a straight frictional substrate is represented as a viscous contractile gel of fixed length $L$. The mechanisms fixing the cell length (see Ref. [15]) are not described here; this simplification is made to make the analysis more transparent.

The time-dependent free boundaries of the cell are $x_{f}(t)$ for the front and $x_{r}(t)=x_{f}(t)-L$ for the rear. The motion of the geometric center of the cell is described by the function $S(t)=\left[x_{r}(t)+x_{f}(t)\right] / 2$. For convenience, the actual position $x \in\left[x_{r}(t), x_{f}(t)\right]$ of a point inside the cell will be replaced in what follows by the comoving coordinate $y(x, t)=x-S(t) \in$ $[-L / 2, L / 2]$.

Momentum balance for the cytoskeleton meshwork with a frictional substrate requires that

$$
\partial_{y} \sigma+f_{e}=\xi w
$$

where $\sigma(y, t)$ is the axial stress field, $w(y, t)$ is the velocity of the gel in the comoving frame of reference, $\xi$ is a friction coefficient, and $f_{e}(y, t)$ is an external force field. The resultant applied traction is therefore

$$
F_{e}(t)=\int_{-L / 2}^{L / 2} f_{e}(y, t) d y
$$

The constitutive behavior of the viscocontractile gel is described by the rheological relation

$$
\sigma=\eta \partial_{y} w+\chi c
$$

where $\eta$ is the gel viscosity, $\chi$ is the contractility, and $c(y, t)$ is the concentration of motors generating the active stress.

Since the segment boundaries are impermeable to the gel, they are propelled at the common but unknown velocity,

$$
V(t)=\dot{S}(t)=\dot{x}_{f}(t)=\dot{x}_{r}(t)=w( \pm L / 2, t),
$$

where the superimposed dot denotes the time derivative. The reaction stress at the two boundaries $\sigma( \pm L / 2, t)=\sigma^{b}(t)$ is a kinematic variable to be determined using the fixed length constraint.

The molecular motors are advected with the flow and undergo a diffusive motion characterized by the flux $J$ so that the motor conservation law can be written as

$$
\partial_{t} c+\partial_{y}[c(w-V)-J]=0 .
$$

In accordance with Fick's law we assume that $J=D \partial_{y} c$ where $D$ is an effective [1,32] diffusion coefficient. The additional drift velocity $V$ in (4) is due to the fact that the time derivative is taken at fixed value of $y$ and $\left.\partial_{t}\right|_{x \text { fixed }}=$ $\left.\partial_{t}\right|_{y \text { fixed }}-V \partial_{y}$. Assuming the initial condition $c(y, 0)=c^{0}(y)$ and adopting no flux boundary conditions $\partial_{y} c( \pm L / 2, t)=0$, we obtain that the total amount of motors remains fixed:

$$
M=\int_{-L / 2}^{L / 2} c^{0}(y) d y=\int_{-L / 2}^{L / 2} c(y, t) d y .
$$

\section{B. Thermodynamics}

A detailed study of the thermodynamics of the AS model can be found in Refs. [1,33]. Here we present a simplified analysis in order to compare it with the case of an active particle. Assuming that temperature remains constant the global dissipation in the system $R$ reads

$$
R=P-\dot{\mathrm{E}} \geqslant 0,
$$

where $\mathrm{E}$ is the energy of the system and $P$ is the power of external forces. In view of (1) we can write

$$
P=-\int_{-L / 2}^{L / 2}\left(\xi w-f_{e}\right) w d y
$$

To compute $\dot{\mathrm{E}}$, we need to take into account the chemical reaction supporting the activity of the motors. If $\zeta(y, t)$ is the reaction progress variable, we write (see Ref. [33] for details)

$$
\dot{\mathrm{E}}=-\int_{-L / 2}^{L / 2}\left[J \partial_{y} \mu+A \dot{\zeta}\right] d y
$$

where $A$ is the affinity of the reaction which is a prescribed constant measuring the degree of the nonequilibrium [34] and $\mu(c)$ is the chemical potential of the motors. Under 
these assumptions, we obtain the explicit expression for the dissipation

$$
R=\int_{-L / 2}^{L / 2}\left[\sigma \partial_{y} w+J \partial_{y} \mu+A \dot{\zeta}\right] d y .
$$

We now make the standard assumption [35] $J=l_{33} \partial_{y} \mu$ and introduce a coupling between mechanics and chemistry in the form $\sigma=\eta \partial_{y} w+l_{12} A, \dot{\zeta}=-l_{12} \partial_{y} w+l_{22} A$. A simple way to express the fact that the molecular motors play the role of a catalyst for the reaction is to assume that the related kinetic coefficients are proportional to the concentration $l_{12}=a c$ and $l_{22}=b c$, where $a$ and $b$ are constants. With this assumption, we recover the constitutive relation (2) with $\chi=a A$. A second consequence is the presence of the mechanical feedback to kinetics $\dot{\zeta}=c\left(b A-a \partial_{y} w\right)$. Finally to recover the Fickian diffusion postulated to close the equation (4), we need to assume a linear dependence of the chemical potential in the concentration field $\mu=k c$ and set $D=k l_{33}$. As a result, we obtain

$$
R=\int_{-L / 2}^{L / 2}\left[b A^{2} c+\eta\left(\partial_{y} w\right)^{2}+k D\left(\partial_{y} c\right)^{2}\right] d y \geqslant 0 .
$$

The first term in (7) describes dissipation due to chemical reaction, the second term is the viscous dissipation, and the last term is the contribution due to diffusion.

\section{Nondimensionalization}

We nondimensionalize distances by the hydrodynamic length $\bar{l}=\sqrt{\eta / \xi}$, times by the diffusional time scale $\bar{t}=$ $\bar{l}^{2} / D$, concentrations by $\bar{c}=M / L$, and stresses by $\bar{\sigma}=\xi D$ (and hence forces by $\bar{f}=\bar{\sigma} / \bar{l}$ and velocities by $\bar{w}=\bar{l} / \bar{t}$ ). The ensuing problem depends on the three nondimensional parameters:

$$
\mathcal{L}:=\frac{L}{\bar{l}}, F:=\frac{F_{e}}{\bar{\sigma}}, \text { and } \mathcal{P}:=\frac{M \chi}{\bar{l} \bar{\sigma}} .
$$

These parameters represent, respectively, the length of the segment in the units of the hydrodynamic length (i.e., the length over which a perturbation in the flow propagates before it is damped), the normalized resultant traction force applied to the system (which is generically a function of time), and the normalized contractility of the motors. The problem also depends on the imposed nondimensional force field $f(y, t)=$ $f_{e}(y, t) / F_{e}(t)$ constrained by the condition $\int_{-\mathcal{L} / 2}^{\mathcal{L} / 2} f(y, t)$ $d y=1$.

Although we consider from now on only nondimensional variables, we keep the same notations for the physical variables (i.e., time, space, stress, velocity, and concentration).

\section{Reduction to a single nonlocal equation}

Combining the force balance with the constitutive relation, we obtain the linear equation for the stress

$$
-\partial_{y y} \sigma+\sigma=F \partial_{y} f+(\mathcal{P} / \mathcal{L}) c .
$$

Solving for $\sigma$, we obtain a nonlocal relation

$$
\sigma=(\mathcal{P} / \mathcal{L}) \tilde{\phi} * c+F \tilde{\phi} * \partial_{y} f
$$

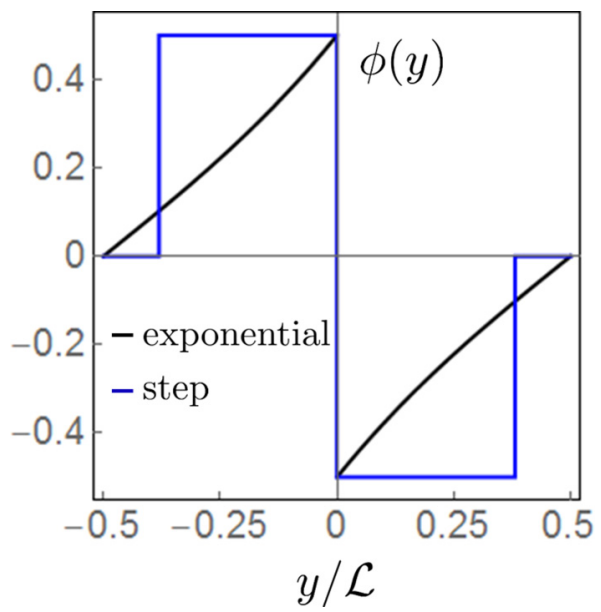

FIG. 1. Two interaction kernels $\phi(y)$ : Black line is the exponential kernel of this paper and blue line is the kernel used in Ref. [36]. Nondimensional length $\mathcal{L}=2$.

where we introduce the notation

$$
\psi * h=\int_{-\mathcal{L} / 2}^{\mathcal{L} / 2} \psi(y-z) h(z, t) d z .
$$

The interaction kernel in (9) is

$$
\tilde{\phi}(z)=\frac{\cosh (z+\mathcal{L} / 2)-2 H(z) \sinh (z) \sinh (\mathcal{L} / 2)}{2 \sinh (\mathcal{L} / 2)},
$$

where $H$ is the Heaviside function. Differentiating the stress and using the force balance equation we obtain the expression for the velocity field

$$
w=(\mathcal{P} / \mathcal{L}) \phi * c+F\left(\phi * \partial_{y} f+f\right),
$$

where $\phi(z)=\partial_{z} \tilde{\phi}(z)$. In Fig. 1 we compare the kernel $\phi$ with the simplified kernel introduced in Ref. [36] on purely topological and symmetry grounds.

Equation (10) may be seen as as the fundamental description of the contraction-driven mechanics: the flow velocity $w$ at point $y$ is induced, first, by the presence in another point $z$ of an active force dipole, represented by a motor concentrationdependent active stress [15,28], and, second, by the passive external force field. We illustrate in Fig. 2 the nonlocal response of the stress and velocity fields to a space-dependent motor or force loading. While a symmetric motor distribution gives rise to a symmetric stress field and an antisymmetric velocity field, the response to a symmetric force field is an antisymmetric stress distribution and a symmetric velocity distribution.

The impenetrability condition (3) can be now used to express the segment velocity

$$
V(t)=\frac{\mathcal{P}}{\mathcal{L}}\{\phi * c\}+F\left\{\phi * \partial_{y} f+f\right\},
$$

where $\{h\}=\left(\left.h\right|_{-\mathcal{L} / 2}+\left.h\right|_{\mathcal{L} / 2}\right) / 2$ denotes the average over the domain boundaries.

To write a single equation for the concentration field we combine (4), (10), and (11) to obtain

$$
\partial_{t} c+\partial_{y}\left\{c\left[(\mathcal{P} / \mathcal{L}) \phi \star c+F\left(\phi \star \partial_{y} f+\delta \star f\right)\right]\right\}=\partial_{y y} c,
$$



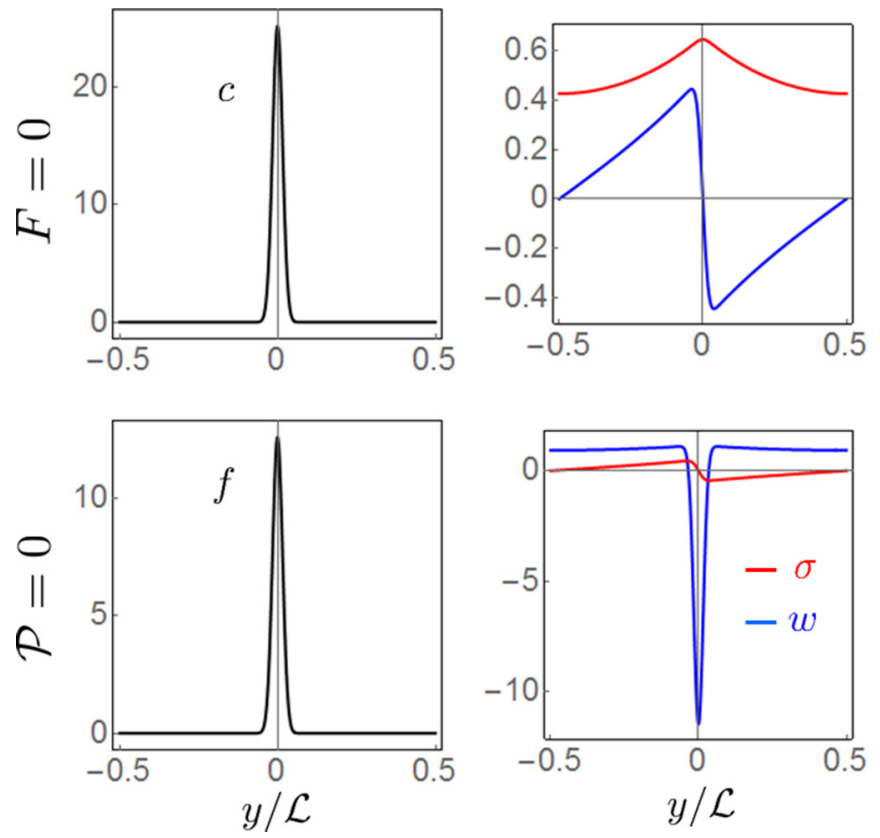

FIG. 2. Nonlocal response of the stress field [see (9)] and the velocity field [see (10)] to a contractility distribution with no external force (first row, $F=0$ and $\mathcal{P}=1$ ) and to a force distribution with no contractility (second row, $\mathcal{P}=0$ and $F=1$ ). Nondimensional length $\mathcal{L}=2$.

where $\delta$ is the Dirac distribution, and we introduce the notation $\phi \star h=\phi * h-\{\phi * h\}$. The no-flux boundary condition then ensures that $\langle c\rangle=1$, where spatial averaging is defined by

$$
\langle h\rangle=\frac{1}{\mathcal{L}} \int_{-\mathcal{L} / 2}^{\mathcal{L} / 2} h(z, t) d z .
$$

In the special case when $F=0$ we obtain the classical KellerSegel model with a quadratic nonlinearity [37]

$$
\partial_{t} c+(\mathcal{P} / \mathcal{L}) \partial_{y}[c \phi \star c]=\partial_{y y} c
$$

After Eq. (12) is solved for $c(y, t)$, the remaining unknowns $\sigma(y, t), w(y, t)$, and $V(t)$ can be reconstructed using Eqs. (9), (10), and (11). Note that the velocity field decouples from the motor concentration field with the latter emerging as the main driver of the overall dynamics.

\section{THE ACTIVE PARTICLE (AP)}

Suppose now that the internal configuration of the motors $c(y, t)$ is not observable and that we have access only to some global polarity measure. We found it convenient to choose it in the form

$$
C(t)=\{\phi * c\} / \mathcal{L},
$$

which is a variable confined to the interval $[-1 / 2,1 / 2]$ and which is nonzero if and only if $c$ is not symmetric (i.e., not even).

\section{A. Model reduction}

To obtain a closed description of the cell dynamics in terms of the "macrovariables" representing the polarity $C(t)$ and the position $S(t)$, we need to project the infinite dimensional active segment (AS) model (12) onto this two-dimensional space defining the active particle (AP) model. To this end, we first average (10) in two different ways. Using (11) we directly obtain

$$
V=\mathcal{P} C+k_{S} F
$$

where

$$
k_{S}=\left\{\phi * \partial_{y} f+f\right\} .
$$

By integrating (10) over space we also obtain

$$
\langle w\rangle=F / \mathcal{L} .
$$

The new macroscopic variable $\langle w\rangle$ naturally enters a macroscopic analog of (4), which we write in the form

$$
\dot{C}+\langle w-V\rangle=-\Phi(C)
$$

Here the term $\langle w-V\rangle$ mimics the drift term in (4). The term on the right-hand side is intended to play the role of diffusion degrading the existing polarity, and therefore the function $\Phi$ is chosen to be increasing and vanishing at $C=0$. We thus write $\Phi(C)=\partial_{C} \bar{E}$, where the potential $\bar{E}$ is convex. In what follows we will be using the expression

$$
\bar{E}(C)=\frac{\alpha}{4} C^{4}+\frac{\mathcal{P}_{c}}{2} C^{2} .
$$

If we now eliminate $\langle w\rangle$ and denote

$$
k_{C}=k_{S}-1 / \mathcal{L}
$$

we obtain the system of ordinary differential equations:

$$
\begin{aligned}
& \dot{S}=\mathcal{P} C+k_{S} F, \\
& \dot{C}=-\partial_{C} E+k_{C} F,
\end{aligned}
$$

where we introduced a new potential $E$ which now contains an active contribution: $\partial_{C} E=\Phi(C)-\mathcal{P} C$. In particular, for the quartic choice of $\bar{E}$ made above, we obtain

$$
E(C)=\frac{\alpha}{4} C^{4}-\frac{\mathcal{P}-\mathcal{P}_{c}}{2} C^{2}
$$

which is a Landau potential with the active term playing a destabilizing role for the symmetric state. The presence of the active term $-\mathcal{P} C^{2} / 2$ ensures that for large $\mathcal{P}$ the potential develops two wells corresponding to two symmetry-related polarized states.

Similar to the AS model, the decoupling of the variable $S$ from the dynamics of the variable $C$ renders the AP model (15) nonpotential: The position of the AP depends on its polarity while the reverse influence is absent.

\section{B. Thermodynamics}

If we multiply $(15)_{2}$ by $\dot{C}$ we find

$$
k_{C} F \dot{C}-\dot{E}=\dot{C}^{2} \geqslant 0 \text {. }
$$

This relation is reminiscent of (5) in the AS model. The terms $k_{C} F \dot{C}$ can be interpreted as the work done by the external 
force $F$ on the collective variable $C$. The rate of change of the energy associated with the variable $C$ is described by the term $\dot{E}$. Finally, the positive definite term $\dot{C}^{2}$ can be associated with dissipation $R$. Note that $\dot{E}$ splits into the sum of a passive term $\dot{\bar{E}}$ representing diffusion and serving the same role as the term $-\int_{-L / 2}^{L / 2} J \partial_{y} \mu d y$ in (6), and the active term $-(\mathcal{P C}) \dot{C}$, representing the internally driven contraction, and playing a role of the analog of the term $-\int_{-L / 2}^{L / 2} A \dot{\zeta} d y$ in (6).

\section{Negative friction coefficient}

Given that the velocity of the particle is essentially enslaved to its polarity, (15) is a direct analog of the RayleighHelmholtz model where the polarity variable is absent and the activity takes the form of a velocity-dependent friction force [31]. Since the dissipation in such a model can be negative, a "friction" term will, in some parameter range, take the form of an "antifriction".

To illustrate this statement in our context it is sufficient to compute the effective frictional viscosity of the AP at zero velocity: $\mu_{0}(\mathcal{P})=\left.\partial_{V} F\right|_{\dot{S}=\dot{C}=0}$. We obtain

$$
\frac{1}{\mu_{0}}=k_{S}+\frac{\mathcal{P}}{\mathcal{P}_{c}-\mathcal{P}} k_{C} .
$$

To highlight the effect, consider the simplest case when $\mathcal{P}_{c} \geqslant$ $\mathcal{P}$ so that $E$ has a single well and (15) has a single steady state $(\dot{S}=\dot{C}=0)$ which is stable. In the case of AS model, the results are similar even though the analysis is less explicit; see Appendix A.

The sign of $\mu_{0}$ in (16) depends on the contractility $\mathcal{P}$ and the constants $k_{S}$ and $k_{C}$, which are functionals of the continuous force distribution. One would expect that always $k_{S} \geqslant 0$ since in the absence of molecular motors $(\mathcal{P}=0)$, a positive resultant force should be able to drag the layer in the forward direction. When $f \geqslant 0$, we have indeed $k_{S} \geqslant 0$ but negativity of $k_{S}$ can still result from a sign-indefinite distribution of external loading; see Appendix B.

In contrast, even when $f \geqslant 0$, the coefficient $k_{C}$ may be negative for some force distributions. A negativity of $k_{C}$ would mean that a positive resultant force favors negative polarity which triggers a competition between the active force $\mathcal{P} C$ and the passive force $k_{S} F$ in determining the AP velocity. On the contrary, a positive value of $k_{C}$ means that a positive value of the resultant force biases the polarity towards a positive value and that the active and passive forces conspire in selecting the velocity.

We start with the simplest situation when the loading is homogeneous $f(y)=1 / \mathcal{L}$. In this case, we obtain $k_{S}=1 / \mathcal{L}$ and $k_{C}=0$. Thus, the coupling between the applied force and the polarity in (15) is absent, and the coefficient $\mu_{0}$ takes its passive value (independent of $\mathcal{P}$ ) which is $\mu_{0}=\mathcal{L}$; see the black line in Fig. 3. This is fully consistent with the behavior of the AS model as in the case of homogeneous loading (12) is independent of the applied forces and reduces to (13): the homogeneous force shifts the flow velocity $w$ only by a constant, pulling the segment as if it were a passive object.

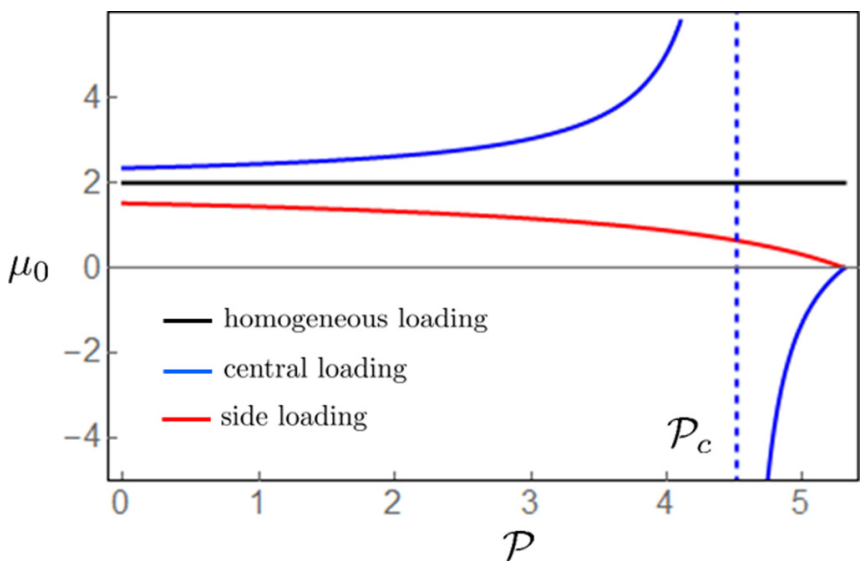

FIG. 3. Effective frictional viscosity $\mu_{0}$ in the AP model as a function of the motor activity $\mathcal{P}$ in the three loading configurations (for the related AS model): homogeneous loading (black line), loading localized in the middle of the segment (blue line), and loading on the segment sides (red line). Nondimensional length $\mathcal{L}=2$.

A more complex case, which was also discussed in Refs. [11,14], is when external forces are applied at the boundaries of the segment (for instance, using cantilevers). Then

$$
f(y)=\beta \delta(y+\mathcal{L} / 2)+(1-\beta) \delta(y-\mathcal{L} / 2),
$$

where $0 \leqslant \beta \leqslant 1$. The configuration of the motors is independent of the partition of the force between the two boundaries (factor $\beta$ ) because the length is fixed and the symmetric part of the loading on the boundary is absorbed into $\sigma^{b}$. Therefore, independently of the value of $\beta$, we obtain

$$
k_{S}(\mathcal{L})=\frac{\operatorname{coth}(\mathcal{L} / 2)}{2} .
$$

In this case $k_{C} \geqslant 0$ and $\mu_{0}$ decreases with the motor activity reaching zero at $\mathcal{P}=\mathcal{P}_{c}$; see the red line in Fig. 3 .

The situation changes radically in the case when the loading is localized in the middle of the segment (say, when a force is applied to the cell nucleus) $f(y)=\delta(y)$. Then $k_{S}=$ $1 /[2 \sinh (\mathcal{L} / 2)]$ and thus $k_{C} \leqslant 0$. Since the coefficient $k_{C}$ is negative, the friction coefficient $\mu_{0}$ increases with the motor activity $\mathcal{P}$ until it blows up and switches sign at the critical value $\mathcal{P}=\mathcal{L} k_{S} \mathcal{P}_{c}$ and then increases again to reach zero at $\mathcal{P}_{c}$; see the blue line in Fig. 3. The fact that the frictional viscosity $\mu_{0}$ can reach zero and even be negative is a feature of many active systems [38,39].

To summarize, the AP model carries a memory of the force distribution in the corresponding AS model, and some particular force distributions may trigger the change of the sign of the effective friction coefficient. In Sec. IV we study the stationary force velocity distribution more systematically, showing, in particular, how the friction coefficient $\mu_{0}$ depends on the parameter $\mathcal{P}$.

In what follows, the force distribution will be always taken in the form (17).

\section{Calibration}

To relate the AP and AS models quantitatively we need to find a relation between the functions $\phi(y)$ and $\Phi(C)$. To 


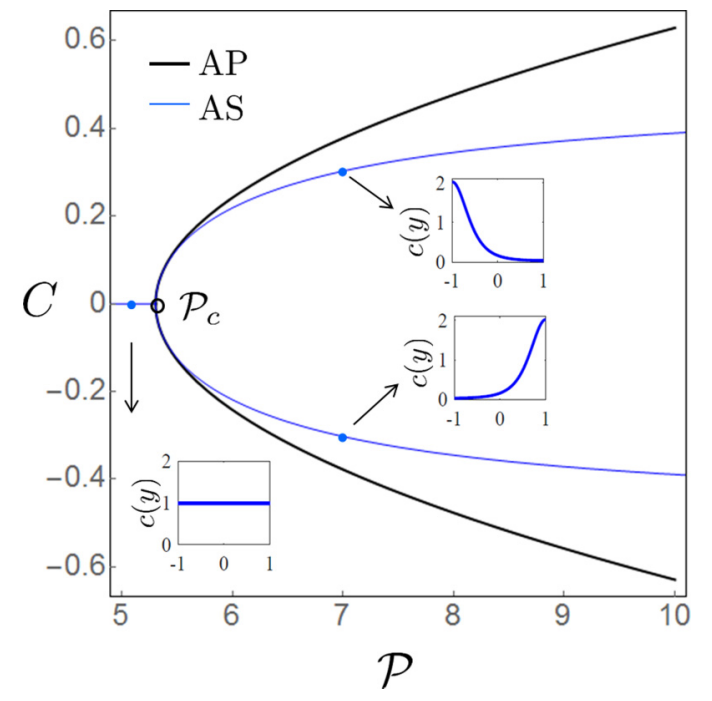

FIG. 4. Spontaneous polarization in AS (thin blue line) and AP (thick black line) models when contractility increases above the critical threshold $\mathcal{P}_{c}$. We show in insets typical concentration profiles of molecular motors along the bifurcated branches in the AS model. Nondimensional length $\mathcal{L}=2$.

do so, it is sufficient to consider the case $F=0$. Under this condition, when the contractility parameter $\mathcal{P}$ in the AS model increases above a critical threshold $\mathcal{P}_{c}(\mathcal{L})$, the symmetric homogeneous solution of (12) $c \equiv 1$ becomes unstable and a polarized motile state emerges as a result of a pitchfork bifurcation (second-order phase transition) leading to two symmetric configurations with opposite polarities [33]. The structure of the bifurcation is shown in Fig. 4, and the expression of $\mathcal{P}_{c}(\mathcal{L})$ is given in Appendix C, see also Fig. 5. To reproduce the same bifurcation in the framework of the AP model (at $F=0$ ), we need to find the minima of the Landau potential $E(C)$. It has

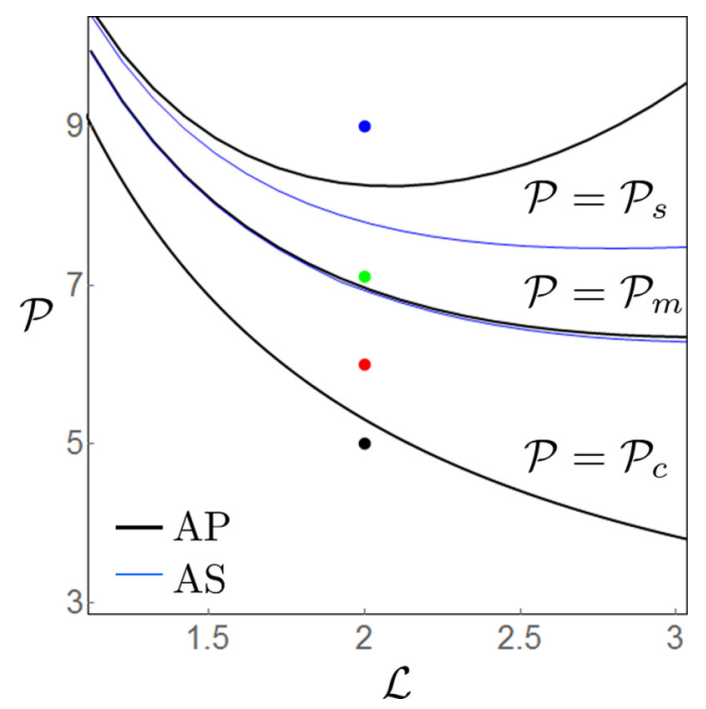

FIG. 5. Dependence of three contractility thresholds $\mathcal{P}_{c}, \mathcal{P}_{m}$, and $\mathcal{P}_{s}$ on the nondimensional length $\mathcal{L}$ for the AP (thick black line) and AS (thin blue line). The value of $\mathcal{P}_{c}$ is the same in both the AS and AP models by construction. The color dots are choices of parameters related to the V-F relations shown in Fig. 6. a single minimum at $C=0$ when $\mathcal{P}<\mathcal{P}_{c}$ and two symmetric minima at $C= \pm \sqrt{\left(\mathcal{P}-\mathcal{P}_{c}\right) / \alpha}$ when $\mathcal{P}>\mathcal{P}_{c}$; see Fig. 4 . The coefficient $\alpha$ is fixed by matching the asymptotic behavior for the two models at $\mathcal{P}=\mathcal{P}_{c}$. From a normal form analysis of the AS model, we obtain $\alpha=\mathcal{P}_{c}^{2} \mathcal{L}^{3} \theta_{2}(\mathcal{L}) / 2$; the analytical expression for the function $\theta_{2}(\mathcal{L})>0$ is given in Appendix $\mathrm{C}$.

The last parameter that needs to be specified to fully define the AP model is $k_{S}(\mathcal{L})$, which encapsulates the external loading distribution. As we have already mentioned, in this paper, we will focus on an external loading from the sides of the segment [see (17)] leading to the expression (18) for $k_{S}$.

The AP model is now fully defined and connected to the AS model by $(15)$ with $\Phi(C), \mathcal{P}_{c}(\mathcal{L}), \alpha(\mathcal{L})$, and $k_{S}(\mathcal{L})$ given above.

The dynamics of both the AP and AS thus depend on two scalar parameters $\mathcal{P}$ and $\mathcal{L}$, characterizing respectively, the activity and the size of the crawler. The overall control is performed by the external loading $F(t)$.

\section{VELOCITY-FORCE RELATIONS}

To test the efficiency of our calibration procedure, we now subject both systems, AS and AP, to a fixed external force and show that the steady-state velocity-force (V-F) relations obtained in Ref. [11] for the AS model can be closely approximated if we use directly the AP model.

In the case of the AS model, we solve numerically Eq. (12) with $\partial_{t} c=0$. In the AP setting we find the stationary value of polarity $C$ directly from the equation $\partial_{C} E=k_{C} F$ and then obtain the V-F relation substituting this value of $C$ into (15). As shown in Fig. 6, both models generate quantitatively similar V-F relations.

When the contractility is sufficiently small, $\mathcal{P}<\mathcal{P}_{c}$ (black curves in Fig. 6), the V-F relations in both models are singlevalued and frictional, meaning that $V F>0$. This is obvious in the AP case since the potential $E(C)$ is convex and the system has only one stable $\left[\partial_{C C} E\left(C_{0}\right)>0\right]$ stationary solution $C_{0}(F)$. The ensuing V-F relation can be written explicitly: $V=k_{S} F+\mathcal{P} C_{0}(F)$.

When contractility becomes large enough, $\mathcal{P}>\mathcal{P}_{c}$ (red, green, and blue curves in Fig. 6), the V-F curves develop a domain of bistability which spreads over a range $F \in\left[-F_{t}, F_{t}\right]$, where, in the AP model, $F_{t}=2\left(\mathcal{P}-\mathcal{P}_{c}\right)^{3 / 2} /\left(3 k_{C} \sqrt{3 \alpha}\right)$. Within this range, the stationary polarity can take three values: $C_{0}^{*}<C_{0}<C_{0}^{* *}$ where $C_{0}^{*}<0<C_{0}^{* *}$ correspond to metastable solutions and $C_{0}$ is an unstable solution $\left[\partial_{C C} E\left(C_{0}\right)<0\right]$. In this range, the V-F relations allow for the coexistence of the two metastable regimes with different signs of velocity: $V^{*}=k_{S} F+\mathcal{P} C_{0}^{*}(F)$ and $V^{* *}=k_{S} F+$ $\mathcal{P} C_{0}^{* *}(F)$. These two branches of the $\mathrm{V}-\mathrm{F}$ relation are connected by the unstable branch $V_{0}=k_{S} F+\mathcal{P} C_{0}(F)$, which is located between the two turning points $F= \pm F_{t}$. Inside the coexistence interval $\left[-F_{t}, F_{t}\right]$, one of the two metastable solutions necessarily operates in an antifrictional regime with $V F \leqslant 0$. Similar bidirectionality is also characteristic of the $\mathrm{V}-\mathrm{F}$ curves describing an ensemble of molecular motors interacting either hydrodynamically [40] or through a rigid backbone [41]. 

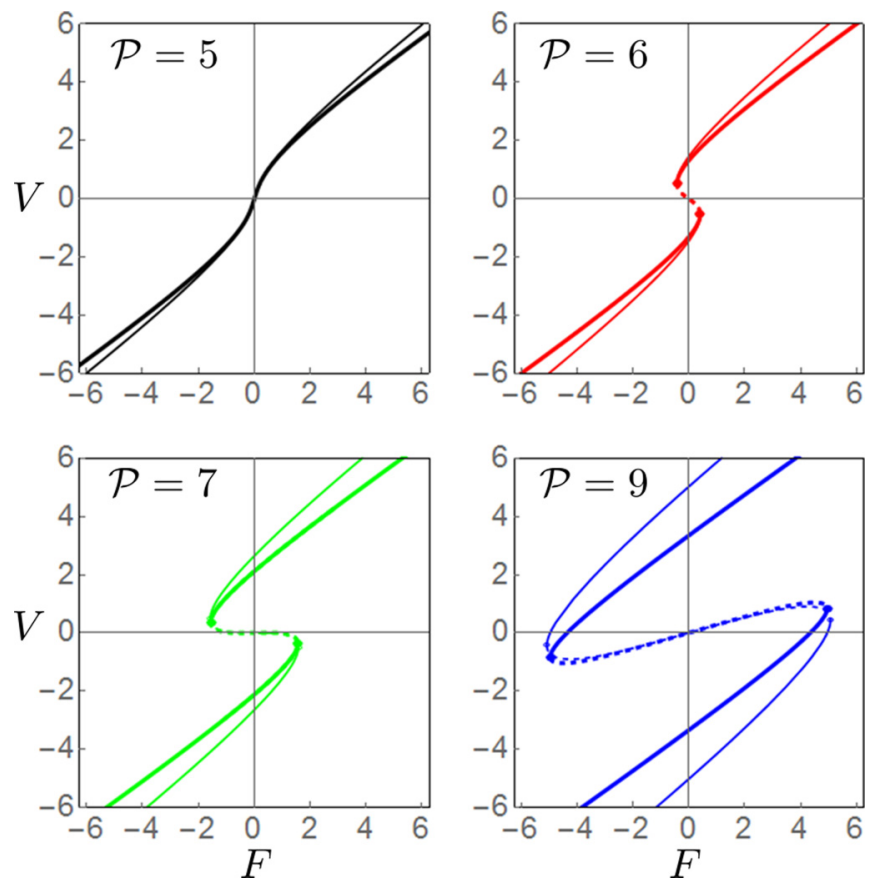

FIG. 6. Comparison of the V-F relations in the AS and AP models. Four typical V-F relations in the AS (thick lines) and the AP (thin lines) models. The dashed parts of the V-F curves correspond to unstable regimes. Parameters $\mathcal{L}=2$ and $\mathcal{P}=5$ (black-upper left corner), $\mathcal{P}=6$ (red-upper right corner), $\mathcal{P}=7$ (green-lower left corner), and $\mathcal{P}=9$ (blue-lower right corner) are represented with the same color dots in Fig. 5.

The most interesting feature of the model is the existence of another threshold, $\mathcal{P}_{m}\left(=k_{S} \mathcal{L} \mathcal{P}_{c}\right.$ in the AP case), beyond which the V-F curves start to display muscle-like stall force states. For $\mathcal{P}_{m}<\mathcal{P}<\mathcal{P}_{s}$ (green curves in Fig. 6), where $\mathcal{P}_{s}=$ $2 k_{S} \mathcal{P}_{c} /\left(3 / \mathcal{L}-k_{S}\right)$ in the AP case, such states are unstable but for $\mathcal{P}>\mathcal{P}_{s}(\mathcal{L})$ (blue curves in Fig. 6) they stabilize. The functions $\mathcal{P}_{m, s}(\mathcal{L})$ for the AS model are compared with those for the AP model in Fig. 5, and the corresponding V-F curves can be read off in Fig. 6. As we illustrate in the schematic Fig. 7, the V-F relations can display a standard hysteresis in force (when $\mathcal{P}_{c}<\mathcal{P}<\mathcal{P}_{s}$ ) or be double hysteretic in both force and velocity (when $\mathcal{P}>\mathcal{P}_{s}$ ). In the latter case, not only two steady-state velocities can be compatible with the same
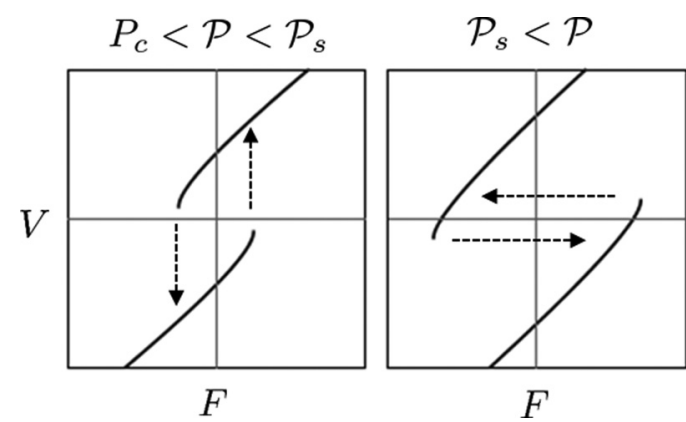

FIG. 7. Schematic representation of the single and double hysteretic V-F relations.

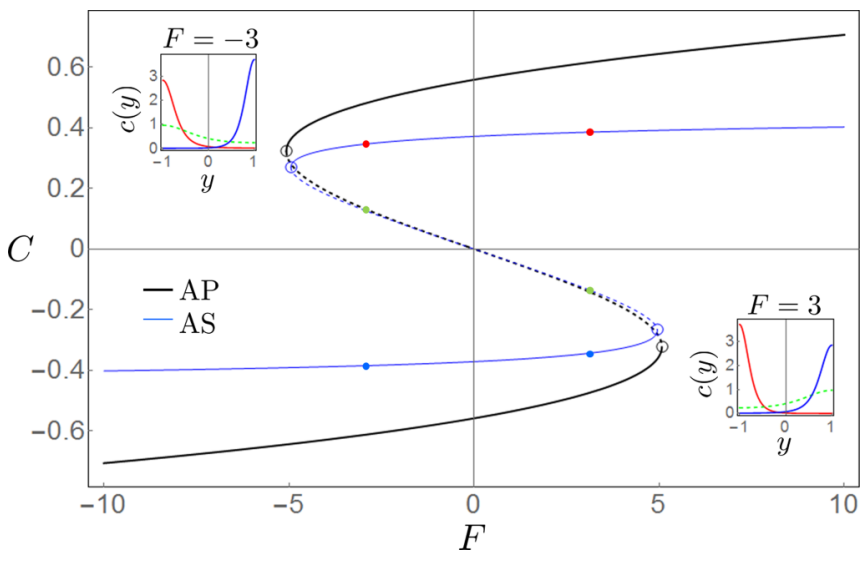

FIG. 8. Comparison of the global polarity $C$ as a function of the force in AS (thin blue) and AP models (thick black). The dashed parts of the C-F curve correspond to unstable regimes. We show in the inset the motor concentration in the AS case at forces $F=-3$ and $F=3$. The red profiles have a positive polarity, and the blue profiles have a negative polarity. A central symmetry transforms a red (resp. blue) profile at a positive force into a blue (resp. red) profile at a negative force. The green profiles are related to the unstable branch. Nondimensional length and contractility: $\mathcal{L}=2$ and $\mathcal{P}=9$.

loading but also two force distributions can be compatible with the same velocity.

Note that in both the AS and AP models, the relation linking the global polarity measure $C$ to the velocity and the force is the same:

$$
C=\left(V-k_{S} F\right) / \mathcal{P} \text {. }
$$

We illustrate in Fig. 8 how $C$ varies as a function of $F$ and how the underlying concentrations of molecular motors change in the AS model. When the loading increases beyond the turning points located at $\pm F_{t}$, the global polarity changes sign as the local motor concentration abruptly switches from one edge of the segment to the other. Similar hysteretic effects have also been found between the angular velocity and the applied torque in a Couette cell containing a polar active gel [42].

Given the good agreement between the AS and AP models in predicting the steady-state regimes, in the rest of the paper we focus on the non-steady-state dynamic behavior of the AP model in two paradigmatic cases when the AP is exposed to either a fluidlike viscous or a solidlike elastic environment. We also compare the nonsteady behavior predicted by the AP and the AS models in the same conditions.

\section{VISCOUS ENVIRONMENT}

Assume that the external force is proportional to the particle velocity

$$
F(t)=-\eta_{\mathrm{p}} \dot{S}(t)
$$

where $\eta_{\mathrm{p}}$ is the (nondimensional) viscosity of the environment. System (15) then takes the form

$$
\left(1+\eta_{\mathrm{p}} k_{S}\right) \dot{S}=\mathcal{P} C, \dot{C}=-\partial_{C} E(C)-\eta_{\mathrm{p}} k_{C} \dot{S} .
$$




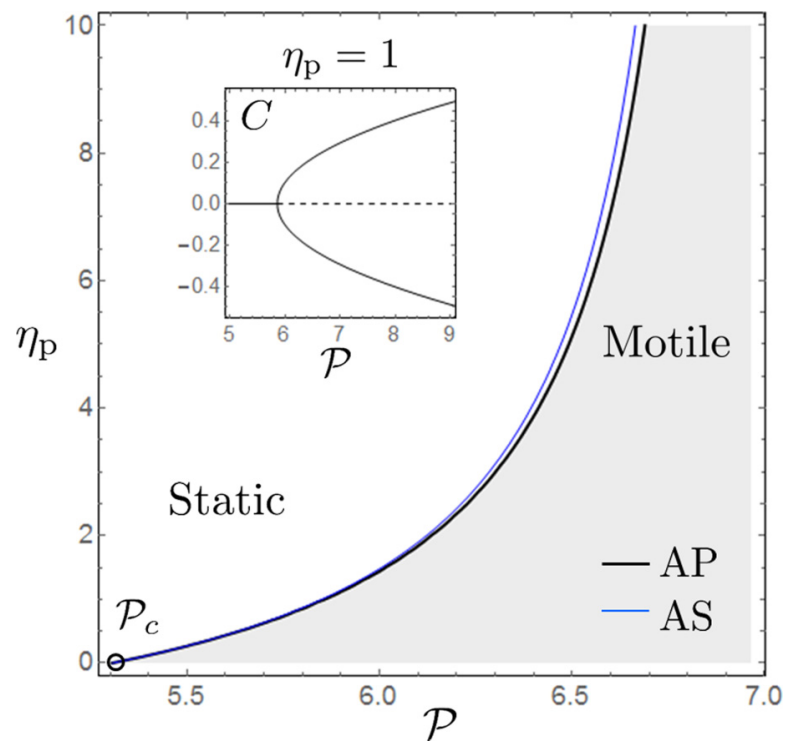

FIG. 9. Phase diagram of an AP subjected to a viscous drag force. The inset shows that the bifurcation from a static to a motile case at a critical contractility remains of second order (supercritical). Such a transition can be also obtained by reducing the environment viscosity. The phase boundary obtained for the AS is superimposed in thin blue. Nondimensional length $\mathcal{L}=2$.

We can reduce (19) to a single nonlinear ordinary differential equation for the particle polarity $\dot{C}=-\partial_{C} E_{\text {eff }}(C)$ where

$$
E_{\text {eff }}(C)=\alpha \frac{C^{4}}{4}-\left[\mathcal{P}\left(1-\frac{\eta_{\mathrm{p}} k_{C}}{1+\eta_{\mathrm{p}} k_{S}}\right)-\mathcal{P}_{c}\right] \frac{C^{2}}{2} .
$$

The viscosity of the environment therefore redresses the onset of motility to the value

$$
\mathcal{P}_{c}^{\text {eff }}=\frac{\mathcal{P}_{c}}{1-\frac{\eta_{\mathrm{p}} k_{C}}{1+\eta_{\mathrm{p}} k_{S}}} \geqslant \mathcal{P}_{c} .
$$

The resulting motility initiation phase diagram is shown in Fig. 9. The effect is the same in the AS case with $\mathcal{P}_{c}^{\text {eff }}$ analytically given in Appendix $\mathrm{C}$ and presented for comparison in Fig. 9 (thin blue line). Note that the bifurcation from a static to motile state as the activity of the motors increases remains supercritical; see inset of Fig. 9.

Interestingly, the same type of transition (static to motile) is also initiated by reducing the environment viscosity. However, $\mathcal{P}_{c}^{\text {eff }}\left(\eta_{\mathrm{p}}\right)$ has an asymptote $\left(=\mathcal{P}_{m}\right.$ for both the AP and AS) when $\eta_{\mathrm{p}} \rightarrow \infty$. This is an indication that the transition threshold $\mathcal{P}_{c}^{\text {eff }}$ depends weakly on the external viscosity when the latter is sufficiently large even though the velocity of the particle remains sensitive to it. The robustness of the threshold suggests that, in this range of parameters, active crawlers can effectively adapt their degree of polarization to the external viscosity.

In Fig. 10 we illustrate the nonsteady motility initiation while comparing the AP and AS dynamic models. One can see that depending on the value of the environmental viscosity the same statically equilibrated initial state can be stable or not: in more viscous environments active agent remains static, while in less viscous environments it (a)

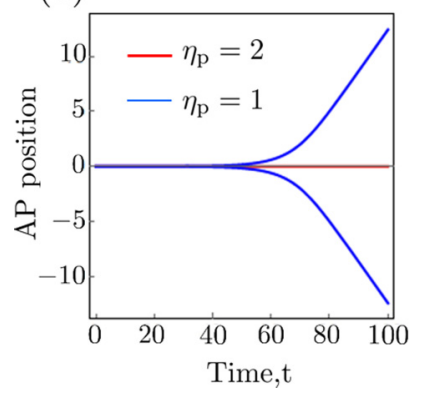

(b)

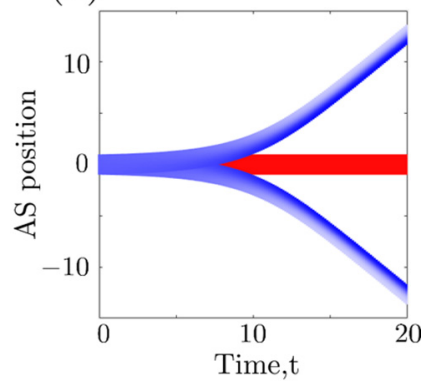

FIG. 10. Initiation of motility in a viscous environment. (a) AP position $S$ as a function of time with two external viscosities corresponding a static (red) and a motile (blue) case. One of the two symmetric trajectory is chosen according to a small bias in the initial polarity. (b) Same type of dynamics for the AS model. The intensity of the coloring is proportional to the level of motor concentration. Nondimensional contractility and length $\mathcal{P}=6$ and $\mathcal{L}=2$.

spontaneously starts to move in one of the two symmetric directions.

\section{ELASTIC CONFINEMENT}

We now consider the case when the environmental force $F(t)$ is given by

$$
F(t)=-k_{\mathrm{p}} S(t),
$$

where $k_{\mathrm{p}}$ is the (nondimensional) stiffness of the confining environment; see Fig. 11. While we have previously investigated this situation numerically in the case of the AS model [11], we now show that the AP model allows one to understand the stability properties of such systems analytically.

Inside a harmonic trap the active agent cannot move persistently, but it can still exhibit a rich dynamics. In the case of the AP model, the main system (15) takes the form

$$
\dot{S}=\mathcal{P} C-k_{\mathrm{p}} k_{S} S, \dot{C}=-\partial_{C} E(C)-k_{\mathrm{p}} k_{C} S,
$$

which combines into the second-order system for the polarity variable

$$
k_{\mathrm{p}} \ddot{C}+\left[k_{\mathrm{p}} \partial_{C C} E(C)+k_{S}\right] \dot{C}+k_{S} \partial_{C} E(C)+k_{C} \mathcal{P} C=0 .
$$

Inspection of Eq. (22) shows that it reduces to a classical Van der Pol equation if $k_{S}=0$. The critical points of (21) are $\left(S_{0}, C_{0}\right)=(0,0)$, corresponding to the force-free static configuration, while the points

$$
\left(S_{s}^{ \pm}, C_{s}^{ \pm}\right)= \pm \frac{\sqrt{k_{S}\left(\mathcal{P}-\mathcal{P}_{c}\right)-k_{C} \mathcal{P}}}{\sqrt{k_{S} \alpha}}\left(\frac{\mathcal{P}}{k_{\mathrm{p}} k_{S}}, 1\right)
$$

describe two symmetrically stalled configurations with the spring under either tension or compression. The linear stability of such states is determined by solving the characteristic

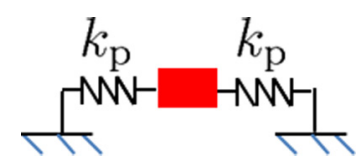

FIG. 11. Scheme of an elastically confined AP (in red). 


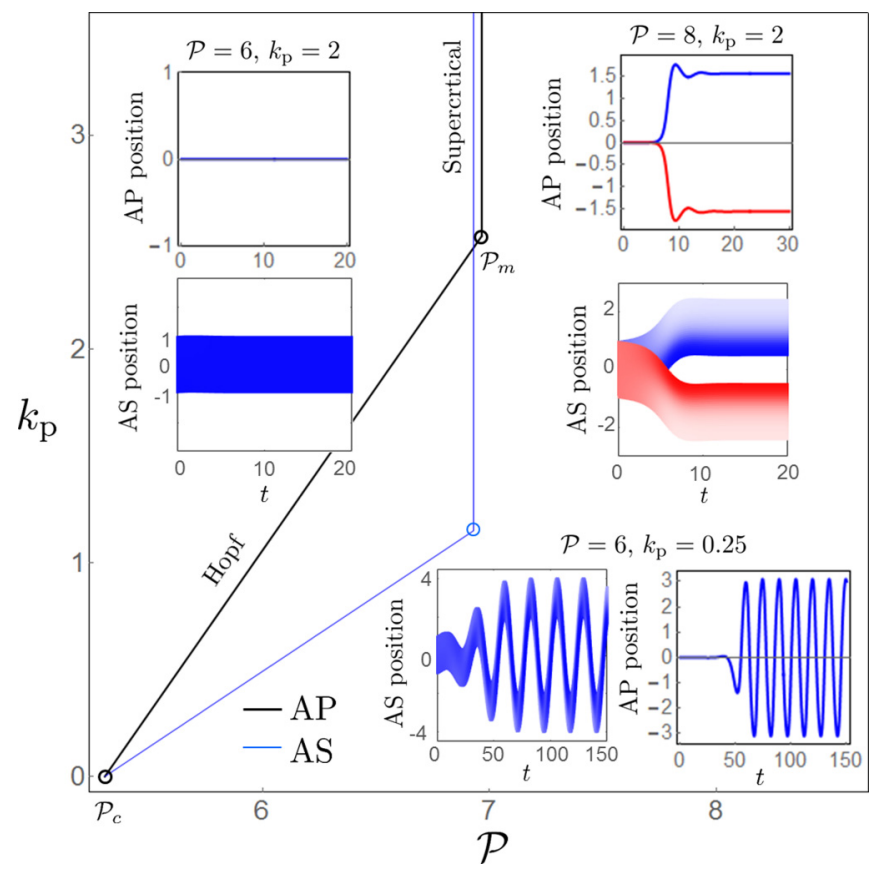

FIG. 12. Linear instability thresholds of the static solution in the $\mathrm{AP}$ and AS case. We show in insets some typical static, stalled, and oscillatory dynamics for the AP and AS case. The intensity of the coloring is proportional to the motor concentration for the AS case. Nondimensional length $\mathcal{L}=2$.

equation

$$
\operatorname{det}\left(\begin{array}{cc}
-k_{S} k_{p}-\omega & \mathcal{P} \\
-k_{C} k_{p} & -3 \alpha C^{2}+\mathcal{P}-\mathcal{P}_{c}-\omega
\end{array}\right)=0
$$

for $C=C_{0}$ and $C=C_{s}^{ \pm}$and finding conditions when the real part of $\omega$ becomes positive.

From such an analysis, we find that the loss of linear stability of the trivial static configuration $\left(S_{0}, C_{0}\right)$ can be of two types depending on the rigidity of the external environment:

(1) If $k_{\mathrm{p}} \leqslant k_{\mathrm{p}}^{*}=k_{C} \mathcal{L} \mathcal{P}_{c} / k_{S}$, the configuration $\left(S_{0}, C_{0}\right)$ stops being linearly stable as a result of a Hopf bifurcation taking place at $\mathcal{P}=k_{\mathrm{p}}\left(\mathcal{P}_{m}-\mathcal{P}_{c}\right) / k_{\mathrm{p}}^{*}+\mathcal{P}_{c}$.

(2) If $k_{\mathrm{p}} \geqslant k_{\mathrm{p}}^{*}$, the configuration $\left(S_{0}, C_{0}\right)$ stops being linearly stable through a supercritical pitchfork bifurcation taking place at $\mathcal{P}=\mathcal{P}_{m}$.

We present in Fig. 12 the comparison of these two linear instabilities for the AP and AS models. The insets illustrate the typical static, stalled and oscillatory regimes. A similar picture emerges from the study of the linear stability of the stalled solutions $\left(S_{s}^{ \pm}, C_{s}^{ \pm}\right)$:

(1) If $k_{\mathrm{p}} \leqslant k_{\mathrm{p}}^{*}$, the configuration $\left(S_{s}^{ \pm}, C_{s}^{ \pm}\right)$stops being linearly stable through a Hopf bifurcation taking place at $\mathcal{P}=\mathcal{P}_{s}-k_{\mathrm{p}}\left(\mathcal{P}_{s}-\mathcal{P}_{m}\right) / k_{\mathrm{p}}^{*}$.

(2) If $k_{\mathrm{p}} \geqslant k_{\mathrm{p}}^{*}$, the configuration $\left(S_{s}^{ \pm}, C_{s}^{ \pm}\right)$stops being linearly stable through a supercritical pitchfork bifurcation taking place at $\mathcal{P}=\mathcal{P}_{m}$.

Both linear stability results are summarized for the AP model on the synthetic phase diagram shown in Fig. 13. In addition, we show there the numerically constructed nonlinear stability boundaries for all three types of solutions: static, stalled, and oscillatory. Interestingly, the supercritical

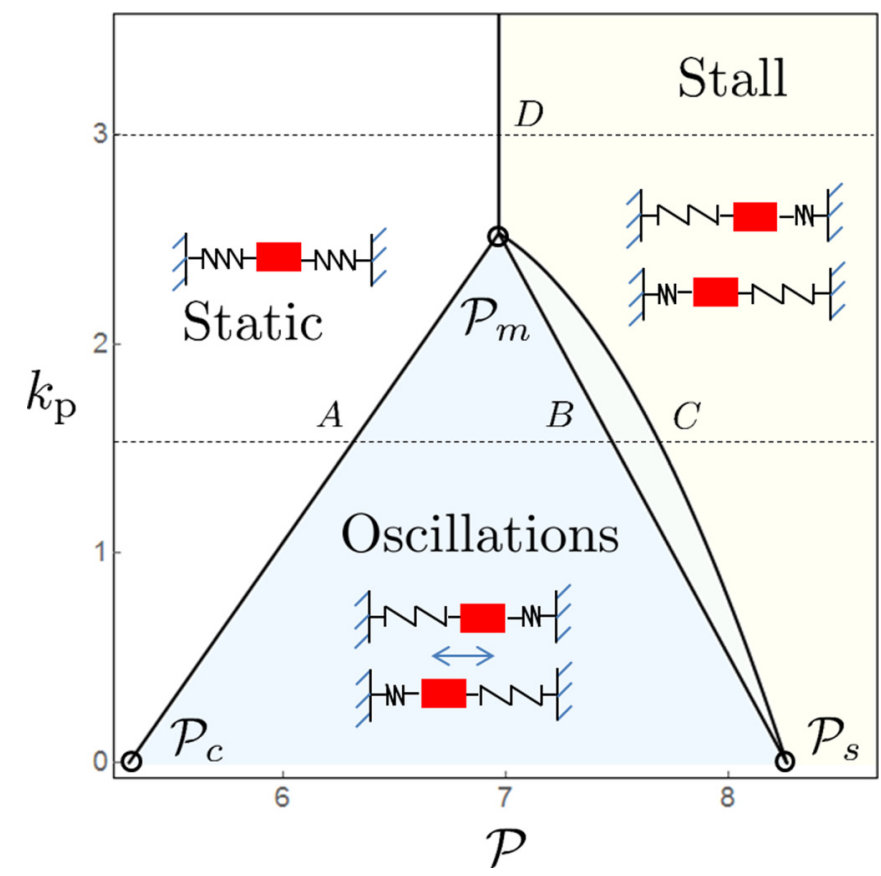

FIG. 13. Stability diagram of an AP confined by harmonic springs depending on the contractility and environment stiffness. Note the region of coexistence (metastability) between the oscillations and stall phases where the two types of solutions coexist. The two thin dashed lines and associated capital letters are related to the bifurcations diagrams shown in Fig. 14. Nondimensional length $\mathcal{L}=2$.

transition from a static to a stalled state becomes insensitive to $k_{\mathrm{p}}$ above the threshold $k_{\mathrm{p}}^{*}$. This indicates again that the AP can self-adapt to the environmental stiffness in order to maintain the same motor activity threshold. We also report the opening of a domain of metastability where oscillatory solutions coexist with stall solutions which we were not able to capture numerically for the AS model in Ref. [11]. This is potentially important as it can open the possibility of complex stop-and-go dynamics for an elastically confined AP subjected to noise.

The origin of such metastability can be understood by reconstructing the global structure of the bifurcation diagram using a numerical continuation method [43]. Typical results are illustrated in Fig. 14. When the environment stiffness is smaller than the tricritical point value, $k_{\mathrm{p}} \leqslant k_{\mathrm{p}}^{*}$ [see Fig. 14(a)], the branch of oscillatory solutions emerging from the static branch reaches a turning point (denoted by $\mathrm{C}$ in Fig. 13 and Fig. 14). As the contractility $\mathcal{P}$ increases beyond this point the oscillatory solutions cease to be stable and the system abruptly switches to the stalled configuration. The same discontinuous transition is associated with the decrease of contractility when the stalled configurations undergoes a Hopf bifurcation at the critical value of $\mathcal{P}$ (denoted by B in Fig. 13 and Fig. 14). The oscillatory and stalled configurations have therefore a domain of metastable coexistence. When $k_{\mathrm{p}} \geqslant k_{\mathrm{p}}^{*}$ [see Fig. 14(b)], this complexity disappears as we observe only a continuous transition from a static to a stalled state (point D in Fig. 13 and Fig. 14). 

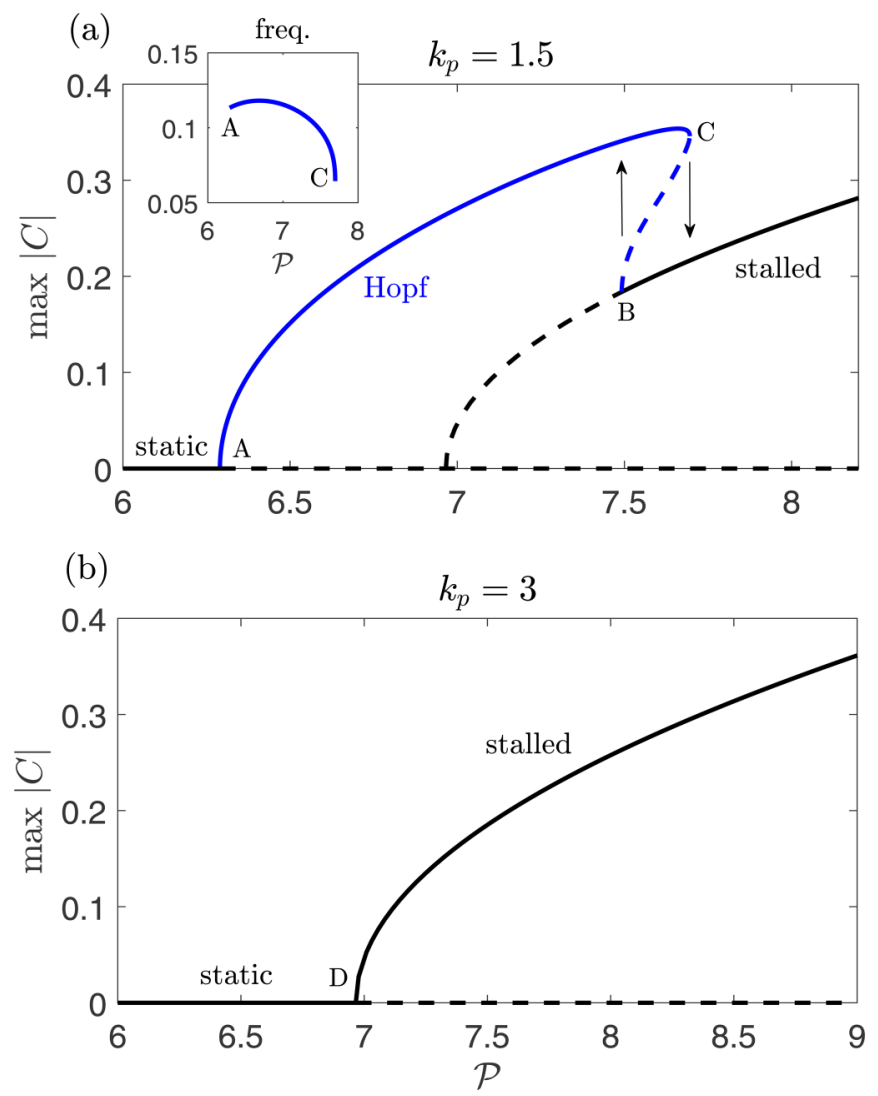

FIG. 14. Two typical bifurcation diagrams for an elastically confined AP when the stiffness $k_{\mathrm{p}}<k_{\mathrm{p}}^{*}$ (a) and when $k_{\mathrm{p}}>k_{\mathrm{p}}^{*}$ (b). The continuation of the Hopf bifurcation is shown in blue, while supercritical pitchforks (second-order phase transitions) are shown in black. In the inset of (a), we show the frequency (inverse of the period) of the stable Hopf oscillations as a function of the continuation parameter. The dashed lines are linearly unstable branches, and full lines are stable. Arrows indicate the discontinuous transitions. The capital letters are related to the phase diagram shown in Fig. 13. Nondimensional length $\mathcal{L}=2$.

To illustrate the structure of the oscillations we show in Fig. 15 the limit-cycle-type regimes for the AP model using the force-velocity coordinates. The parameters are chosen in the oscillatory phase, see Fig. 13. As the stiffness of the environment gets smaller, the oscillations amplitude and their period are increasing, which results, in the limit $k_{\mathrm{p}} \rightarrow 0$, in an almost steady-state behavior as the limit cycle is now adequately described by the hysteretic V-F relation obtained in Sec. IV.

Oscillations driven by molecular motors are ubiquitous across various space and time scales in cell biology $[44,45]$. Cell shape oscillations are often shown to be resulting from a periodic regulation by signaling molecules (Rho GTPases) controlling the motors' contractility [46]. Indeed, the activation or inhibition dynamic between several Rho GTPases can form an autonomous clock acting as a pacemaker [47]. However, center-of-mass oscillations of living cells, associated with periodic reversals of the molecular motors polarity, were also repeatedly observed $[48,49]$. There exist theoretical models of cell motility involving both cytoskeleton contrac- (a)

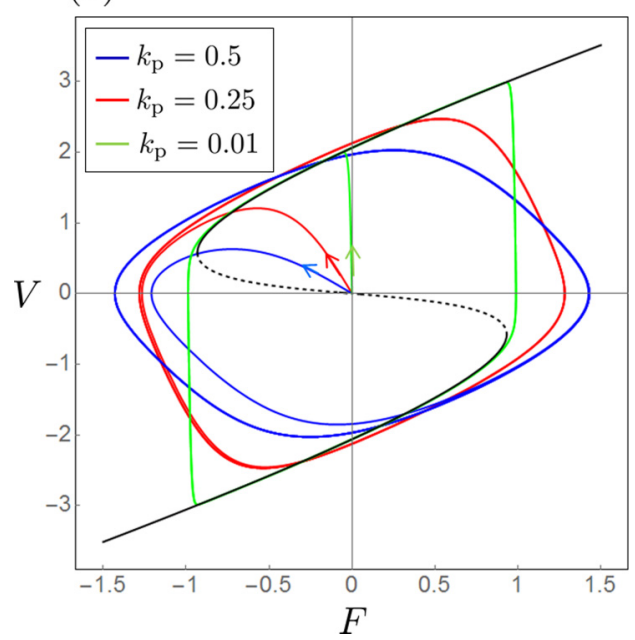

(b)
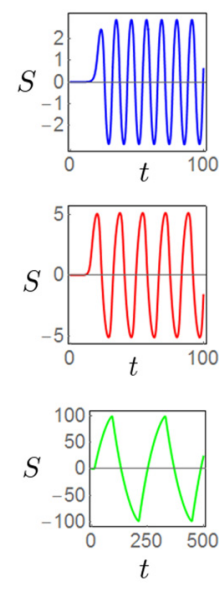

FIG. 15. Dynamics of the oscillations for the stiffness $k_{\mathrm{p}}$ of the environment starting from the initial state $\left(S_{0}, C_{0}\right)$. (a) The trajectories of the oscillating particle in the phase space $\left(k_{\mathrm{p}} S, \dot{S}\right)$ for three different stiffnesses. We superimpose in black the V-F curve obtained for a fixed force (Sec. IV). (b) The related particle position steady-state oscillations. Nondimensional length and contractility $\mathcal{L}=2$ and $\mathcal{P}=6.5$.

tion and protrusion aiming at capturing the emergence of such oscillations. For instance, in Ref. [50], oscillations emerge from a coupling between cell shape and biochemical polarization, while in Ref. [51], they result from a delay between actin and myosin flow in the cell cortex. Here we report that oscillations can also spontaneously arise simply from the mechanical interaction of the cell with its elastic environment. Interestingly, oscillations similar to the ones found in this paper were also reported at a smaller scale where a bead-tailed actin filament propelled by the collective action of myosin motors was tethered to an optical trap [52].

To complement this analysis, we now briefly discuss the case of a breakable environmental confinement from where the active agent can escape. To this end we assume that $F(t)=-k_{\mathrm{p}} S(t) H\left[l_{\mathrm{p}}-|S(t)|\right]$, where the parameter $l_{\mathrm{p}}$ characterizes the (nondimensional) breaking limit of the confining spring; see Fig. 16. In this case, the AP can break out of the confinement and reach a motile state. We show in Fig. 17 the resulting phase diagram at a given value of $l_{\mathrm{p}}$. The AP can now become motile as soon as the spring reaches the

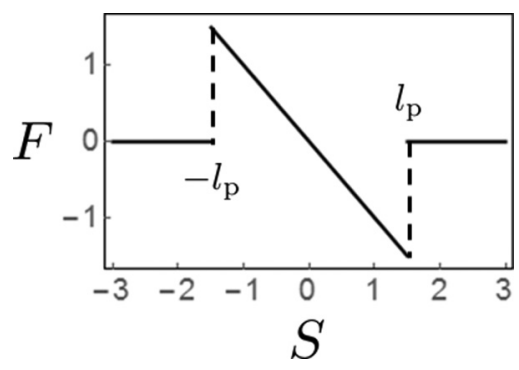

FIG. 16. Schematic representation of the harmonic confinement with a breaking threshold. 


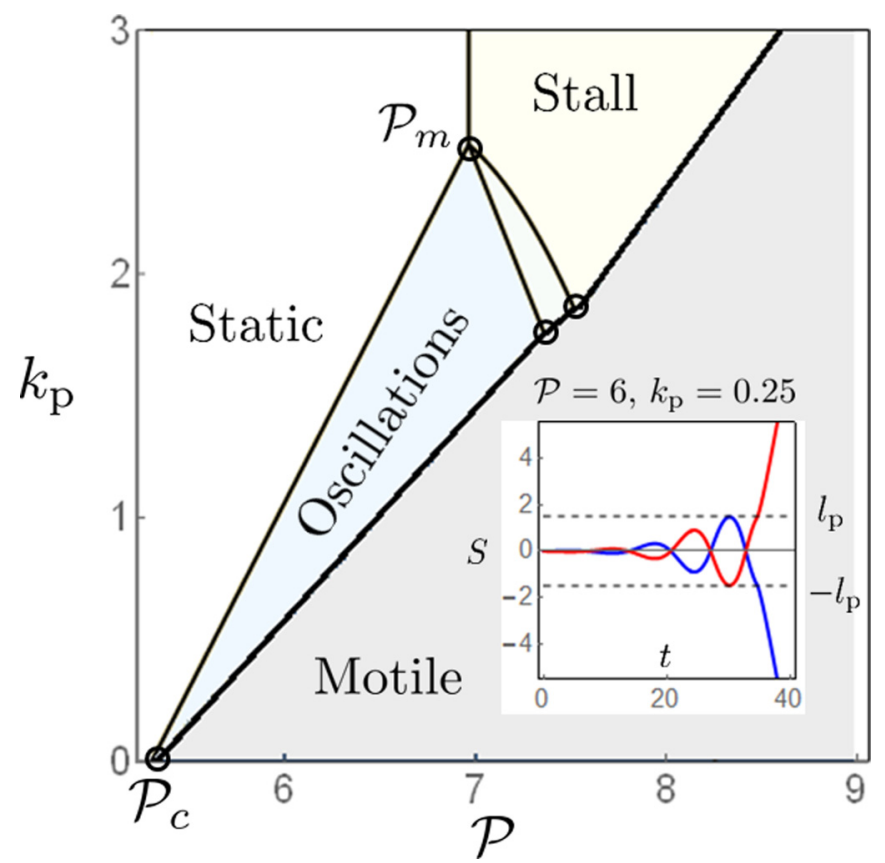

FIG. 17. Stability diagram of an AP confined by breakable harmonic springs. The position of the boundary of the motile phase depends on the initial conditions of (15), which are here taken to be $(S, C)(t=0)=\left(S_{0}, C_{0}\right)$. We show in the inset some typical dynamic of the AP breaking out of the harmonic confinement. Nondimensional length and breaking limit $\mathcal{L}=2$ and $l_{\mathrm{p}}=1.5$.

elongation $l_{\mathrm{p}}$ with a nonzero speed. Such an escape scenario is reminiscent of an epithelial to mesenchymal transition where cells break out from the confinement of their neighbors and start to move persistently on their own.

\section{CONCLUSIONS}

Starting with a one-dimensional model of contractiondriven crawling we developed an active particle model. Such a particle is able to adjust its polarity to the applied force. Both models generate quantitatively similar force-velocity relations which can describe hysteresis in velocity and force. In the presence of a viscous resistance from the environment, the obtained model predicts the emergence of polarity and the associated initiation of motility when the viscosity is reduced. If elastically confined, both the active segment and particle can develop dynamical oscillations. The model suggests that there exists a domain of parameters where oscillatory and stalled states coexist, which suggests the possibility of stochastic switch between the two regimes. We can anticipate even more complex dynamic attractors in a viscoelastic environment of Kelvin-Voigt or Maxwell type and/or when the external rheology becomes nonlinear involving, for instance, plastic deformations.

\section{ACKNOWLEDGMENTS}

P.R. acknowledges support from a CNRS-Momentum grant. T.P. was supported by the EPSRC Engineering Nonlinearity Project No. EP/K003836/1. L.T. is grateful to the French government, which supported his work under Grant No. ANR-10-IDEX-0001-02 PSL.

\section{APPENDIX A: EFFECTIVE VISCOSITY}

When the applied force is much larger than the contractile force $F \gg \mathcal{P}$, Eq. (11) furnishes the explicit steady-state velocity-force $(\mathrm{V}-\mathrm{F})$ relation: $V=(F / 2) \operatorname{coth}(\mathcal{L} / 2)$. The inverse of the slope of the $\mathrm{V}-\mathrm{F}$ relation at zero force (i.e., the effective frictional viscosity) can then be computed directly:

$$
\mu_{\infty}=2 \tanh (\mathcal{L} / 2) .
$$

In the opposite case, when the external forces are negligible $\mathcal{P} \gg F$, the homogeneous solution $c \equiv 1$ is the stable steady state as long as $\mathcal{P} \leqslant \mathcal{P}_{c}$. By performing a first-order Taylor expansion around this solution for small $F$, we can compute the effective frictional viscosity $\mu_{0}$.

To this end we introduce a small parameter $\epsilon$ and substitute the expansions $c(y)=1+\epsilon c_{1}(y), V=\epsilon V_{1}$, and $F=\epsilon F_{1}$ into Eq. (12). At the first order we obtain the linear integrodifferential equation

$$
\begin{gathered}
\int_{-1 / 2}^{1 / 2}\{\phi[\mathcal{L}(u-v)]-\phi(\mathcal{L} / 2-\mathcal{L} v)\} c_{1}(v) d v \\
+F_{1}[\psi(\mathcal{L} u)-\psi(\mathcal{L} / 2)]=\frac{1}{\mathcal{L}} \partial_{u} c_{1} .
\end{gathered}
$$

Here we have used the rescaled variable $u$ and applied the no-flux boundary conditions. Note that we still need to impose the constraint $\int_{-1 / 2}^{1 / 2} c_{1}(u) d u=0$.

In view of the exponential nature of the kernel, Eq. (A1) can be solved analytically. It can be first transformed into the following system of second-order linear differential equations:

$$
\begin{gathered}
-\frac{1}{\mathcal{L}^{2}} \partial_{u u} \mathbf{X}+\mathbb{M} \mathbf{X}=\mathbf{V}(u), \quad \text { where } \mathbf{X}=\left(\begin{array}{l}
c_{1} \\
\sigma_{1}
\end{array}\right), \\
\mathbf{V}=\left(\begin{array}{c}
-F_{1} \tilde{\psi}(\mathcal{L} u) \\
0
\end{array}\right) \text { and } \mathbb{M}=\left(\begin{array}{ll}
-\mathcal{P} / \mathcal{L} & 1 \\
-\mathcal{P} / \mathcal{L} & 1
\end{array}\right) .
\end{gathered}
$$

The boundary conditions take the form

$$
\begin{aligned}
& \left.\partial_{u} c_{1}\right|_{-1 / 2}=0, \int_{-1 / 2}^{1 / 2} c_{1}(u) d u=0,\left.\sigma_{1}\right|_{-1 / 2}=\left.\sigma_{1}\right|_{1 / 2} \\
& \quad \times \text { and }\left.\partial_{u} \sigma_{1}\right|_{-1 / 2}=\left.\partial_{u} \sigma_{1}\right|_{1 / 2} .
\end{aligned}
$$

The solution of this system reads

$$
c_{1}(u)=\frac{\operatorname{csch}(\omega / 2) \sinh (u \omega)-u \omega \operatorname{coth}(\omega / 2)}{2-(\mathcal{P} / \mathcal{L}) \omega \operatorname{coth}(\omega / 2)} F_{1},
$$

where $\omega^{2}=\mathcal{L}^{2}(1-\mathcal{P} / \mathcal{L})$. Finally, the substitution of $c_{1}(u)$ into (11) gives the linear part of the force velocity relation

$$
V_{1}=F_{1}\left(\frac{\omega}{\mathcal{L}}\right)^{3} \frac{\operatorname{coth}(\omega / 2)}{2-(\mathcal{P} / \mathcal{L}) \omega \operatorname{coth}(\omega / 2)} .
$$

In Fig. 18 we show the effective viscosity at zero force $\mu_{0}=\partial F_{1} / \partial V_{1}$ as a function of the parameter $\mathcal{P}$. When $\mathcal{P}=0$, the value $\mu_{0}$ coincides with $\mu_{\infty}$ because the $\mathrm{V}$-F relation is linear over the whole range of forces. As the parameter $\mathcal{P}$ increases, $\mu_{0}$ decreases, which is the signature of the contrac- 


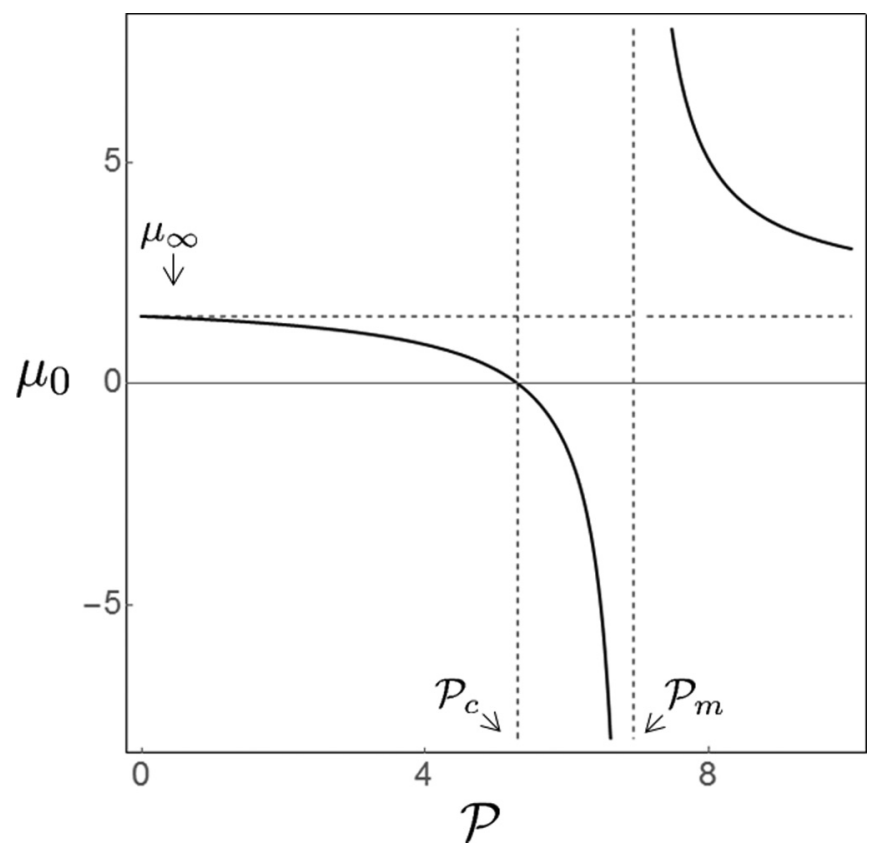

FIG. 18. Effective frictional viscosity at the origin of the VF curve, $\mu_{0}$ as a function of the nondimensional contractility $\mathcal{P}$. Nondimensional length $\mathcal{L}=2$.

tile activity being responsible for the induced flow inside the cell. When $\mathcal{P}$ reaches the value $\mathcal{P}_{c}$, the viscosity $\mu_{0}$ vanishes and becomes negative for $\mathcal{P}>\mathcal{P}_{c}$. The value of $\mu_{0}$ continues to decrease and eventually diverges at $\mathcal{P}=\mathcal{P}_{m}$ indicating a complete flattening of the $\mathrm{V}-\mathrm{F}$ relation close to the origin. If $\mathcal{P}$ increases beyond $\mathcal{P}_{m}$, then $\mu_{0}$ becomes positive again. Note, however, that expression (A4) is obtained as we perturbed the homogeneous solution. Such a solution is the stable attractor of the initial value problem only when $\mathcal{P} \leqslant \mathcal{P}_{c}$, and therefore, the values of $\mu_{0}$ obtained for $\mathcal{P} \geqslant \mathcal{P}_{c}$ are associated with unstable regimes (when the external force is controlled.)

\section{APPENDIX B: NONPOSITIVE DEFINITENESS OF THE COEFFICIENT $\boldsymbol{k}_{S}$}

Using the known expression of the kernel $\phi$, the general expression for the coefficient $k_{S}$, given by (14), can be rewritten in the form

$$
k_{S}=\int_{-\mathcal{L} / 2}^{\mathcal{L} / 2} \frac{\cosh (z)}{2 \sinh (\mathcal{L} / 2)} f(z) d z .
$$

It then clear that if $f(z) \geqslant 0$, then $k_{S} \geqslant 0$. However, if we use the nonsign-definite distributed loading $f$ which takes negative values close to the sides of the segments and a positive value in the center, then the structure of the explicit multiplier in front of $f(z)$ in (B1) suggests that $k_{S}$ can become negative. For instance, if $f(z)=-a[\delta(z+\mathcal{L} / 2)+$
$\delta(z-\mathcal{L} / 2)]+b \delta(z)$ with $a, b \geqslant$ and $b-2 a=1$, then $k_{S} \leqslant 0$ as long as $a \geqslant 1 /\left[4 \sinh (\mathcal{L} / 4)^{2}\right]$.

\section{APPENDIX C: BIFURCATIONS AND NORMAL FORMS}

Consider a steady-state configuration of AS in the absence of an externally applied force $(F=0)$. In this case $\partial_{t} c \equiv 0$, and we can integrate (4) to obtain an expression for $c$ as a function of $\sigma$. Then substituting this expression into (8) leads to the nonlocal boundary value problem (see Ref. [32] for details):

$$
\left\{\begin{array}{c}
-\frac{1}{\mathcal{L}^{2}} \partial_{u u} s(u)+s(u)+s^{b}=\theta\left(\frac{e^{s(u)-v u}}{\int_{-1 / 2}^{1 / 2} e^{s(u)-v u} d u}-1\right) \\
s\left( \pm \frac{1}{2}\right)=0 \text { and } \partial_{u} s\left( \pm \frac{1}{2}\right)=v .
\end{array}\right.
$$

Here we introduced the notations $u=y / \mathcal{L} \in[-1 / 2,1 / 2]$, $\theta \equiv \mathcal{P} / \mathcal{L}, s(u)=\sigma(u)-\sigma^{b}, s^{b}=\sigma^{b}-\theta$, and $v=\mathcal{L} V$. For steady states and $F=0$, Eq. (C1) is equivalent to (12) as $c$ can be reconstructed from $s$ and $v$ using the formula

$$
c(u)=\frac{e^{s(u)-v u}}{\int_{-1 / 2}^{1 / 2} e^{s(u)-v u} d u} .
$$

Equation $(\mathrm{C} 1)$ has the unique homogeneous solution

$$
s=0, v=0 \text { and } s^{b}=0 .
$$

Below we study the bifurcations from this trivial solution giving rise to nontrivial solutions as the parameter $\theta$ increases.

To this end, we choose a small parameter $\epsilon$ and perform a Taylor expansion near the homogeneous solution keeping the terms up to third order:

$$
\left\{\begin{array}{l}
s=0+\epsilon s_{1}+\frac{\epsilon^{2}}{2} s_{2}+\frac{\epsilon^{3}}{6} s_{3}+o\left(\epsilon^{3}\right), \\
v=0+\epsilon v_{1}+\frac{\epsilon^{2}}{2} v_{2}+\frac{\epsilon^{3}}{6} v_{3}+o\left(\epsilon^{3}\right), \\
s^{b}=0+\epsilon s_{1}^{b}+\frac{\epsilon^{2}}{2} s_{2}^{b}+\frac{\epsilon^{3}}{6} s_{3}^{b}+o\left(\epsilon^{3}\right) .
\end{array}\right.
$$

We also write a similar expansion for the bifurcation parameter

$$
\theta=\theta_{0}+\epsilon \theta_{1}+\frac{\epsilon^{2}}{2} \theta_{2}+\frac{\epsilon^{3}}{6} \theta_{3}+o\left(\epsilon^{3}\right) .
$$

Substituting these expansions into $(\mathrm{C} 1)$ and introducing the operator

$$
\begin{aligned}
\mathbb{L}_{\text {in }}\left(s(u), s^{b}, v\right)= & -\frac{\partial_{u u} s(u)}{\mathcal{L}^{2}}+\left(1-\theta_{0}\right) s(u) \\
& +\left(1-\theta_{0}\right) s^{b}+\theta_{0} v u
\end{aligned}
$$

we obtain:

at first order,

$$
\mathbb{L}_{\text {in }}\left(s_{1}, s_{1}^{b}, v_{1}\right)=0,
$$

at second order,

$$
\begin{aligned}
\mathbb{L}_{\text {in }}\left(s_{2}, s_{2}^{b}, v_{2}\right)= & 2\left\{\frac { 1 } { 2 4 } \theta _ { 0 } \left[12 s_{1}(u)\left(s_{1}(u)+2 s_{1}^{b}-2 v_{1} u\right)+24 v_{1} \int_{-1 / 2}^{1 / 2} u s_{1}(u) d u\right.\right. \\
& \left.\left.-12 \int_{-1 / 2}^{1 / 2} s_{1}(u)^{2} d u+24 s_{1}^{b^{2}}-24 s_{1}^{b} v_{1} u+12 v_{1}^{2} u^{2}-v_{1}^{2}\right]+\theta_{1}\left[s_{1}(u)+s_{1}^{b}-v_{1} u\right]\right\},
\end{aligned}
$$


at third order,

$$
\begin{aligned}
\mathbb{L}_{\text {in }}\left(s_{3}, s_{3}^{b}, v_{3}\right)= & \frac{1}{4}\left(\theta _ { 0 } \left\{12 s_{1}(u)\left(2 s_{1}^{b^{2}}+s_{2}^{b}\right)+12 s_{2}(u)\left[s_{1}(u)+s_{1}^{b}-v_{1} u\right]\right.\right. \\
& +12\left[\int_{-1 / 2}^{1 / 2} u s_{1}(u) d u\right]\left[2 v_{1} s_{1}(u)+4 s_{1}^{b} v_{1}-2 v_{1}^{2} u+v_{2}\right]-24 s_{1}^{b} v_{1} u s_{1}(u)+12 s_{1}^{b} s_{1}(u)^{2} \\
& -24 s_{1}^{b}\left[\int_{-1 / 2}^{1 / 2} s_{1}(u)^{2} d u\right]-12 \int_{-1 / 2}^{1 / 2} s_{1}(u) s_{2}(u) d u+12 v_{1}^{2} u^{2} s_{1}(u)-12 v_{1}^{2} \int_{-1 / 2}^{1 / 2} u^{2} s_{1}(u) d u-v_{1}^{2} s_{1}(u) \\
& -12 v_{1} u s_{1}(u)^{2}+12 v_{1} u \int_{-1 / 2}^{1 / 2} s_{1}(u)^{2} d u+12 v_{1} \int_{-1 / 2}^{1 / 2} u s_{1}(u)^{2} d u-12 v_{2} u s_{1}(u)+4 s_{1}(u)^{3}-4 \int_{-1 / 2}^{1 / 2} s_{1}(u)^{3} d u \\
& -12 s_{1}(u)\left[\int_{-1 / 2}^{1 / 2} s_{1}(u)^{2} d u\right]+24 s_{1}^{b^{3}}+u\left(-24 s_{1}^{b^{2}} v_{1}-12 s_{1}^{b} v_{2}-12 s_{2}^{b} v_{1}+v_{1}^{3}\right)+24 s_{1}^{b} s_{2}^{b}-2 s_{1}^{b} v_{1}^{2} \\
& \left.+12 v_{1} u^{2}\left(s_{1}^{b} v_{1}+v_{2}\right)+12 v_{1} \int_{-1 / 2}^{1 / 2} u s_{2}(u) d u-4 v_{1}^{3} u^{3}-v_{1} v_{2}\right\} \\
& +\theta_{1}\left[12 s_{1}(u)\left(s_{1}(u)+2 s_{1}^{b}-2 v_{1} u\right)+24 v_{1} \int_{-1 / 2}^{1 / 2} u s_{1}(u) d u-12 \int_{-1 / 2}^{1 / 2} s_{1}(u)^{2} d u+24 s_{1}^{b^{2}}-12 u\left(2 s_{1}^{b} v_{1}+v_{2}\right)\right. \\
& \left.\left.+12 s_{2}(u)+12 s_{2}^{b}+12 v_{1}^{2} u^{2}-v_{1}^{2}\right]+12 \theta_{2}\left[s_{1}(u)+s_{1}^{b}-v_{1} u\right]\right) .
\end{aligned}
$$

At all orders the boundary conditions remain

$$
s_{i}( \pm 1 / 2)=0 \text { and } \partial_{u} s_{i}( \pm 1 / 2)=v_{i} .
$$

\section{Bifurcation points}

The spectral analysis of (C2) produces a countable number of bifurcation points. Although we provide below a general analysis of all these points, we emphasize that direct numerical simulations of (12) show that the only stable steady-state branches are the trivial branch when $\mathcal{P} \leqslant \mathcal{P}_{c}$ and the first motile branch $D_{1}$ when $\mathcal{P}>\mathcal{P}_{c}$. See Ref. [32] for further details.

Introducing

$$
\omega^{2}=\mathcal{L}^{2}\left(1-\theta_{0}\right)
$$

we obtain in the first order,

$$
\begin{aligned}
s_{1}(u)= & C_{1} \cosh [\omega(u+1 / 2)]+C_{2} \sinh [\omega(u+1 / 2)] \\
& -s_{1}^{b}+v_{1} u\left(\omega^{2}-\mathcal{L}^{2}\right) / \omega^{2} .
\end{aligned}
$$

Note that the solution $\omega=0$ should be excluded because it produces the same homogeneous solution. The four constants $C_{1}, C_{2}, s_{1}^{b}$, and $v_{1}$ follow from the four boundary conditions (C5), which leads to a homogeneous linear system of equations. This algebraic problem has nontrivial solutions when the determinant of the matrix

$$
\left(\begin{array}{cccc}
1 & 0 & -1 & \left(\mathcal{L}^{2} / \omega^{2}-1\right) / 2 \\
0 & \omega & 0 & -\mathcal{L}^{2} / \omega^{2} \\
\cosh (\omega) & \sinh (\omega) & -1 & \left(1-\mathcal{L}^{2} / \omega^{2}\right) / 2 \\
\omega \sinh (\omega) & \omega \cosh (\omega) & 0 & -\mathcal{L}^{2} / \omega^{2}
\end{array}\right)
$$

cancels out, yielding the transcendental characteristic equation

$$
2[\cosh (\omega)-1]+\left(\omega^{2} / \mathcal{L}^{2}-1\right) \omega \sinh (\omega)=0 .
$$

The solutions of this equation split into two families depending on whether parameter $\omega$ is real or purely imaginary. In the first (resp. second) case we denote $\omega_{c}=|\omega| \geqslant 0$ (resp. $\left.\omega_{c}=-|\omega| \leqslant 0\right)$, which leads to

$$
\left\{\begin{array}{cc}
2 \tanh \left(\omega_{c} / 2\right)=\left(1-\omega_{c}^{2} / \mathcal{L}^{2}\right) \omega_{c} & \text { if } \omega_{c} \geqslant 0 \\
2\left[\cos \left(\omega_{c}\right)-1\right]+\left(\omega_{c}^{2} / \mathcal{L}^{2}+1\right) \omega_{c} \sin \left(\omega_{c}\right)=0 & \text { if } \omega_{c} \leqslant 0 .
\end{array}\right.
$$

It is convenient to analyze equations $(\mathrm{C} 9)_{1}$ and $(\mathrm{C} 9)_{2}$ separately:

1. When $\omega$ is real, Eq. (C9) $)_{1}$ has a unique solution provided $2 \sqrt{3} \leqslant \mathcal{L}$. Otherwise, it has no solution. The corresponding eigenvector can be written as

$$
\left(\begin{array}{c}
s_{1}^{b} \\
v_{1} \\
s_{1}(u)
\end{array}\right)=\left(\begin{array}{c}
0 \\
1 \\
\frac{\mathcal{L}^{2}}{\omega_{c}^{3} \cosh \left(\omega_{c} / 2\right)}\left[\sinh \left(u \omega_{c}\right)-2 u \sinh \left(\omega_{c} / 2\right)\right]
\end{array}\right) .
$$

Since $v_{1} \neq 0$ the corresponding bifurcation leads to a motile configuration that we denote $D_{1}$.

2. When $\omega$ is purely imaginary, Eq. (C9) ${ }_{2}$ has two families of solutions:

(a) The first family is explicitly parametrized with an integer $\omega_{c}=-2 m \pi$ with $m \geqslant 1$, and the associated eigenvector reads

$$
\left(\begin{array}{c}
s_{1}^{b} \\
v_{1} \\
s_{1}(u)
\end{array}\right)=\left(\begin{array}{c}
1 \\
0 \\
\cos \left[\omega_{c}(u+1 / 2)\right]-1
\end{array}\right)
$$

Since $v_{1}=0$, the bifurcated solution describes a static cell. We denote this family $S_{m}$. 


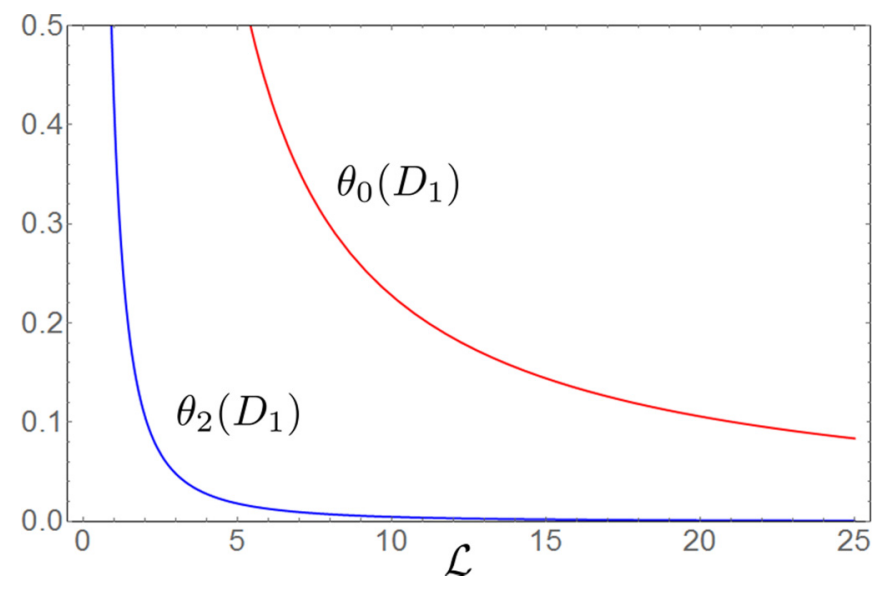

FIG. 19. Eigenvalues $\theta_{0}$ and $\theta_{2}$ as a function of $\mathcal{L}$ for the first motile branch $D_{1}$.

(b) The second family consists of a countable set of negative roots of the equation

$$
2 \tan \left(\omega_{c} / 2\right)=\left(1+\omega_{c}^{2} / \mathcal{L}^{2}\right) \omega_{c} .
$$

The largest root exists only if $\mathcal{L} \leqslant 2 \sqrt{3}$, and the corresponding eigenvector reads

$$
\left(\begin{array}{c}
s_{1}^{b} \\
v_{1} \\
s_{1}(u)
\end{array}\right)=\left(\begin{array}{c}
0 \\
1 \\
\frac{-\mathcal{L}^{2}}{\omega_{c}^{3} \cos \left(\omega_{c} / 2\right)}\left[\sin \left(u \omega_{c}\right)-2 u \sin \left(\omega_{c} / 2\right)\right]
\end{array}\right) .
$$

Since $v_{1} \neq 0$, these roots of the characteristic equation are associated with motile branches. We denote this family $D_{m}$ with $m \geqslant 1$.

The critical bifurcation threshold $\mathcal{P}_{c}$ introduced in the main text can be written as $\mathcal{P}_{c}=\mathcal{L} \theta_{0}\left(D_{1}\right)$ where the relation between $\theta_{0}$ and $\omega_{c}$ follows from (C6). See also Fig. 19.

In the presence of an external viscous friction $\eta_{\mathrm{p}}$, the Dirichlet boundary conditions in $(\mathrm{C} 1)$ are modified into

$$
s\left( \pm \frac{1}{2}\right)=\mp \frac{\eta_{\mathrm{p}} v}{2 \mathcal{L}},
$$

which modifies the critical contractility value controlling the onset of motility into $\mathcal{P}_{c}^{\text {eff }}\left(\mathcal{L}, \eta_{\mathrm{p}}\right)$ as $(\mathrm{C} 9)_{1}$ and $(\mathrm{C} 10)$, respectively, become

$$
2 \tanh \left(\omega_{c} / 2\right)=\left[1-\omega_{c}^{2} / \mathcal{L}^{2}\left(1+\eta_{\mathrm{p}} / \mathcal{L}\right)\right] \omega_{c}
$$

and

$$
2 \tan \left(\omega_{c} / 2\right)=\left[1+\omega_{c}^{2} / \mathcal{L}^{2}\left(1+\eta_{\mathrm{p}} / \mathcal{L}\right)\right] \omega_{c} .
$$

The resulting value of $\mathcal{P}_{c}^{\text {eff }}$ is shown in Fig. 9.

\section{Normal forms}

Each bifurcation is now characterized by the eigenvalue $\theta_{0}$ and the eigenvector $\left[s_{1}(u), s_{1}^{b}, v_{1}\right]$. This information is not sufficient to find the shape of the bifurcated branch close to a bifurcation point. To this end we need to use higher-order equations (C3)-(C4).

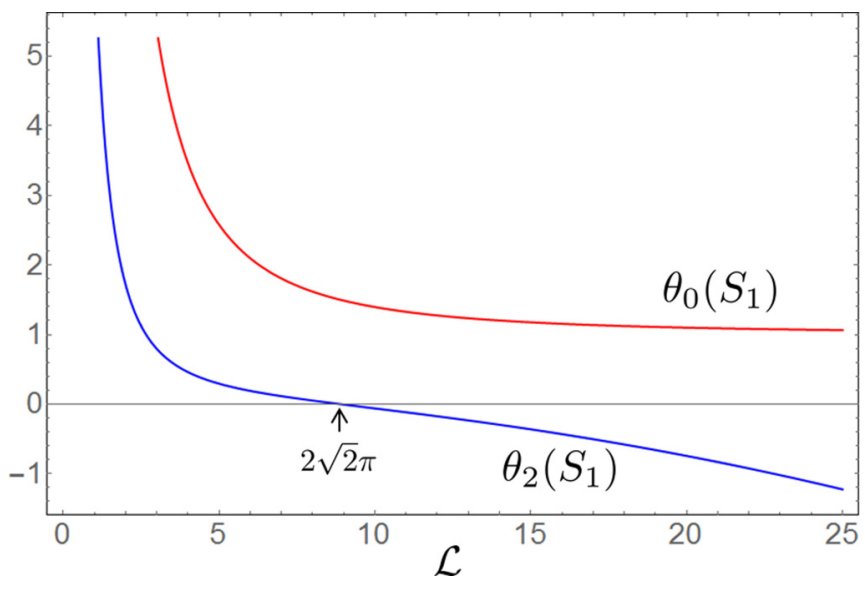

FIG. 20. Eigenvalues $\theta_{0}$ and $\theta_{2}$ as a function of $\mathcal{L}$ for the first static branch $S_{1}$.

Starting from second order, the right-hand side of Eq. (C3) must be in the range of the operator $\mathbb{L}_{i n}$. This is equivalent (Fredholm alternative) to the requirement that this expression is orthogonal to the kernel of the dual of $\mathbb{L}_{\text {in }}$. In our case, this property reduces to imposing an orthogonality condition in the space $\left(C_{1}, C_{2}, s^{b}, v\right)$ with the kernel of the transpose of (C8). The resulting scalar equation sets the value of $\theta_{1}$.

For both static and motile branches, we find $\theta_{1}=0$, which means that the static and motile bifurcations are of pitchfork type. The super- or subcritical nature of the bifurcation follows from third order. Solving first (C3) with $\theta_{1}=0$ leads to the solution $\left[s_{2}^{b}, v_{2}, s_{2}(u)\right]$, whose detailed expression is not given here.

We can now apply the same analysis as above to Eq. (C4), which gives, for the motile branches:

$$
\begin{aligned}
\theta_{2}= & \left\{( \mathcal { L } ^ { 2 } - \omega ^ { 2 } ) \left[\mathcal{L}^{10}\left(3 \omega^{2}+770\right)\right.\right. \\
& -6 \mathcal{L}^{8}\left(2 \omega^{4}+215 \omega^{2}+1540\right) \\
+ & 6 \mathcal{L}^{6}\left(4 \omega^{4}+85 \omega^{2}+660\right) \omega^{2}-2 \mathcal{L}^{4}\left(15 \omega^{2}+79\right) \omega^{6} \\
+ & \left.\left.21 \mathcal{L}^{2}\left(\omega^{2}+8\right) \omega^{8}-6 \omega^{12}\right]\right\} / \\
& \left\{144 \mathcal{L}^{2} \omega^{8}\left[-\mathcal{L}^{4}+2 \mathcal{L}^{2}\left(\omega^{2}+6\right)-\omega^{4}\right]\right\} .
\end{aligned}
$$

This expression is always positive indicating that all motile bifurcations are supercritical. We illustrate in Fig. 19 the eigenvalues $\theta_{0}$ and $\theta_{2}$ as a function of the length parameter $\mathcal{L}$ for the first motile branch $D_{1}$.

To complete the picture, a similar but simpler analysis for the static branches can be carried out. Given that the expression for $\theta_{0}$ can be given explicitly, we can compute

$$
\theta_{2}=\frac{1}{48}\left(\frac{32 \pi^{2} m^{2}}{\mathcal{L}^{2}}-\frac{\mathcal{L}^{2}}{\pi^{2} m^{2}}+4\right)
$$

This value is not always positive, which indicates that the pitchfork bifurcation can be super- or subcritical depending on the value of $m$ and $\mathcal{L}$. In Fig. 20 we illustrate the dependence of $\theta_{0}$ and $\theta_{2}$ on $\mathcal{L}$ for the first static branch $S_{1}(m=1)$. 
[1] I. S. Aronson (Ed.), Physical Models of Cell Motility (Springer, Berlin, 2016).

[2] B. Alberts, A. Johnson, J. Lewis, M. Raff, K. Roberts, and P. Walter, Molecular Biology of the Cell, 4th ed. (Garland Science, New-York, 2002).

[3] W. R. Holmes, J. Park, A. Levchenko, and L. Edelstein-Keshet, PLoS Comput. Biol. 13, e1005524 (2017).

[4] J. Park, W. R. Holmes, S. H. Lee, H.-N. Kim, D.-H. Kim, M. K. Kwak, C. J. Wang, L. Edelstein-Keshet, and A. Levchenko, Proc. Natl. Acad. Sci. USA 114, E5750 (2017).

[5] C. Huang, K. Jacobson, and M. D. Schaller, J. Cell Sci. 117, 4619 (2004).

[6] L. Lara Rodriguez and I. C. Schneider, Integr. Biol. 5, 1306 (2013).

[7] F. Ziebert and I. S. Aronson, PloS ONE 8, e64511 (2013).

[8] T. Iskratsch, H. Wolfenson, and M. P. Sheetz, Nat. Rev. Mol. Cell Biol. 15, 825 (2014).

[9] R. A. Desai, S. B. Gopal, S. Chen, and C. S. Chen, J. R. Soc., Interface 10, 20130717 (2013).

[10] E. Scarpa, A. Roycroft, E. Theveneau, E. Terriac, M. Piel, and R. Mayor, Biol. Open 2, 901 (2013).

[11] P. Recho, T. Putelat, and L. Truskinovsky, New J. Phys. 21, 033015 (2019).

[12] B. Merchant, L. Edelstein-Keshet, and J. J. Feng, Development. Biol. 444, S262 (2018).

[13] D. A. Kulawiak, B. A. Camley, and W.-J. Rappel, PLoS Comput. Biol. 12, e1005239 (2016).

[14] P. Recho and L. Truskinovsky, Phys. Rev. E 87, 022720 (2013).

[15] T. Putelat, P. Recho, and L. Truskinovsky, Phys. Rev. E 97, 012410 (2018).

[16] A. Mogilner, J. Math. Biol. 58, 105 (2009).

[17] F. Jülicher, K. Kruse, J. Prost, and J.-F. Joanny, Phys. Rep. 449, 3 (2007).

[18] B. Rubinstein, M. F. Fournier, K. Jacobson, A. B. Verkhovsky, and A. Mogilner, Biophys. J. 97, 1853 (2009).

[19] D. Shao, W.-J. Rappel, and H. Levine, Phys. Rev. Lett. 105, 108104 (2010).

[20] F. Ziebert, S. Swaminathan, and I. S. Aronson, J. R. Soc., Interf. 9, 1084 (2011).

[21] L. Giomi and A. DeSimone, Phys. Rev. Lett. 112, 147802 (2014).

[22] E. Tjhung, A. Tiribocchi, D. Marenduzzo, and M. Cates, Nat. Commun. 6, 5420 (2015).

[23] T. Vicsek, A. Czirók, E. Ben-Jacob, I. Cohen, and O. Shochet, Phys. Rev. Lett. 75, 1226 (1995).

[24] A. Peshkov, E. Bertin, F. Ginelli, and H. Chaté, Eur. Phys. J.: Spec. Top. 223, 1315 (2014).

[25] M. C. Marchetti, J.-F. Joanny, S. Ramaswamy, T. B. Liverpool, J. Prost, M. Rao, and R. A. Simha, Rev. Mod. Phys. 85, 1143 (2013).

[26] B. A. Camley and W.-J. Rappel, J. Phys. D 50, 113002 (2017).
[27] V. Hakim and P. Silberzan, Rep. Prog. Phys. 80, 076601 (2017).

[28] P. Recho, T. Putelat, and L. Truskinovsky, Phys. Rev. Lett. 111, 108102 (2013).

[29] E. Barnhart, K.-C. Lee, G. M. Allen, J. A. Theriot, and A. Mogilner, Proc. Natl. Acad. Sci. USA 112, 5045 (2015).

[30] P. Maiuri, E. Terriac, P. Paul-Gilloteaux, T. Vignaud, K. McNally, J. Onuffer, K. Thorn, P. A. Nguyen, N. Georgoulia, D. Soong et al., Curr. Biol. 22, R673 (2012).

[31] P. Romanczuk, M. Bär, W. Ebeling, B. Lindner, and L. Schimansky-Geier, Eur. Phys. J.: Spec. Top. 202, 1 (2012).

[32] P. Recho, T. Putelat, and L. Truskinovsky, J. Mech. Phys. Solids 84, 469 (2015).

[33] P. Recho, J.-F. Joanny, and L. Truskinovsky, Phys. Rev. Lett. 112, 218101 (2014).

[34] J. Prost, F. Jülicher, and J.-F. Joanny, Nat. Phys. 11, 111 (2015).

[35] S. R. De Groot and P. Mazur, Non-equilibrium Thermodynamics (Dover, New York, 1984).

[36] K. Kruse and F. Jülicher, Phys. Rev. Lett. 85, 1778 (2000).

[37] E. F. Keller and L. A. Segel, J. Theor. Biol. 30, 225 (1971).

[38] B. M. Haines, I. S. Aranson, L. Berlyand, and D. A. Karpeev, Phys. Biol. 5, 046003 (2008).

[39] H. M. López, J. Gachelin, C. Douarche, H. Auradou, and E. Clément, Phys. Rev. Lett. 115, 028301 (2015).

[40] P. Malgaretti, I. Pagonabarraga, and J.-F. Joanny, Phys. Rev. Lett. 119, 168101 (2017).

[41] F. Jülicher and J. Prost, Phys. Rev. Lett. 75, 2618 (1995).

[42] S. Fürthauer, M. Neef, S. W. Grill, K. Kruse, and F. Jülicher, New J. Phys. 14, 023001 (2012).

[43] E. J. Doedel, H. B. Keller, and J. P. Kernevez, Int. J. Bifurcation Chaos 1, 493 (1991).

[44] K. Kruse and D. Riveline, in Forces and Tension in Development, edited by M. Labouesse, Current Topics in Developmental Biology Vol. 95 (Academic Press, San-Diego, 2011), pp. 67-91.

[45] G. B. Blanchard, J. Étienne, and N. Gorfinkiel, Curr. Opin. Genet. Dev. 51, 78 (2018).

[46] M. Nishikawa, S. R. Naganathan, F. Jülicher, and S. W. Grill, Elife 6, e19595 (2017).

[47] X. Qin, E. Hannezo, T. Mangeat, C. Liu, P. Majumder, J. Liu, V. Choesmel-Cadamuro, J. A. McDonald, Y. Liu, B. Yi et al., Nat. Commun. 9, 1210 (2018).

[48] I. Lavi, M. Piel, A.-M. Lennon-Duménil, R. Voituriez, and N. S. Gov, Nat. Phys. 12, 1146 (2016).

[49] A. Godeau, Ph.D. thesis, University of Strasbourg (2016).

[50] B. A. Camley, Y. Zhao, B. Li, H. Levine, and W.-J. Rappel, Phys. Rev. Lett. 111, 158102 (2013).

[51] A. Farutin, J. Etienne, C. Misbah, and P. Recho, Phys. Rev. Lett. 123, 118101 (2019).

[52] P.-Y. Plaçais, M. Balland, T. Guérin, J.-F. Joanny, and P. Martin, Phys. Rev. Lett. 103, 158102 (2009). 